\title{
A 3-D MULTIPLEX PAPER-MICROFLUIDIC PLATFORM
}

\author{
A Thesis \\ presented to \\ the Faculty of California Polytechnic State University, \\ San Luis Obispo
}

\author{
In Partial Fulfillment \\ of the Requirements for the Degree \\ Master of Science in Biomedical Engineering
}

by

Mitchell Patrick Young

September 2016 
(C) 2016

Mitchell Patrick Young

ALL RIGHTS RESERVED 


\section{COMMITTEE MEMBERSHIP}

TITLE: A 3-D Multiplex Paper-Microfluidic Platform

AUTHOR: Mitchell Patrick Young

DATE SUBMITTED: September 2016

COMMITTEE CHAIR: David Clague, Ph.D.

Professor of Biomedical Engineering

COMMITTEE MEMBER: $\quad$ Scott Hazelwood, Ph.D.

Professor of Biomedical Engineering

COMMITTEE MEMBER: Lanny Griffin, Ph.D.

Professor of Biomedical Engineering 


\author{
ABSTRACT \\ A 3-D Multiplex Paper-Microfluidic Platform \\ Mitchell Patrick Young
}

3-D paper-based microfluidic devices (micoPADs) are small and portable devices made out of paper that offer a promising platform for diagnostic applications outside of a laboratory. These devices are easy to use, low cost, require no power source, and capable of detecting multiple targets simultaneously. The work in this thesis demonstrated the ability of a 3-D paper-microfluidic platform to simultaneously detect 5 targets. Rubber cord stock was used in conjunction with an acrylic housing unit to apply pressure along the edge of the channel. The indirect pressure application was successful in promoting vertical fluid flow between layers. Average channel development times were recorded between 110 seconds and 150 seconds.

The implementation of the 3-D paper-microfluidic platform as a diagnostic device was validated with a colorimetric glucose assay. In a novel application, reagents were deposited onto the 3-D platform via a glucose reagent pencil created by Martinez et al. A visual signal was observed for the successful detection of glucose at a concentration of 1.2 mM. These results offer promise for future work in combing new reagent deposition techniques with a multi-layer paper-microfluidic platform. Overall, this research made advancements in the design of a paper-microfluidic platform capable of the simultaneous detection of 5 targets.

Keywords: Paper-microfluidics, microPAD, multiplex, glucose reagent pencil 


\section{ACKNOWLEDGMENTS}

I would like to thank my parents, Tom and Sherri Young, for all of their support as I worked on both my undergraduate and graduate degrees at Cal Poly. Without your help I would not have been put in such a great position to succeed. I would also like to thank Dr. Clague for all the help and advice given as I worked on my thesis. Without your guidance I would not have had the success I did with my work. Lastly, I would like to thank all the other members of the microfluidics group that took time to help me through some of the issues I encountered. It was always a relief knowing I could turn to one of you for help. 


\section{TABLE OF CONTENTS}

Page

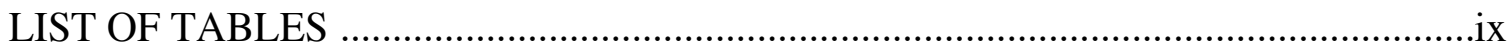

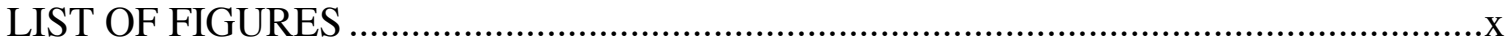

CHAPTER

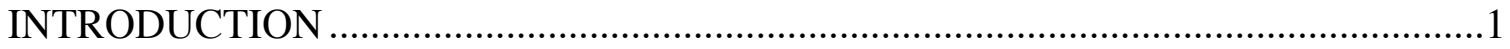

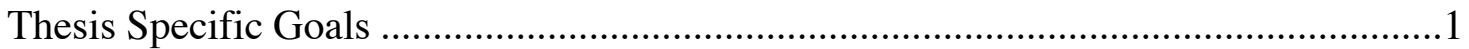

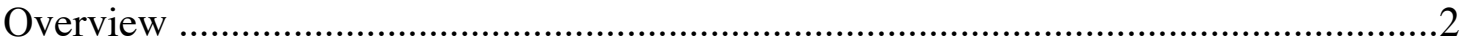

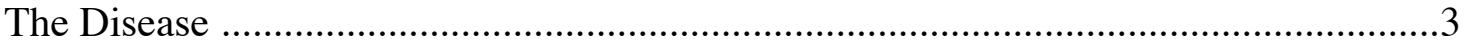

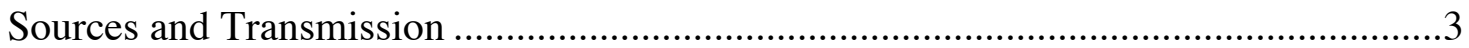

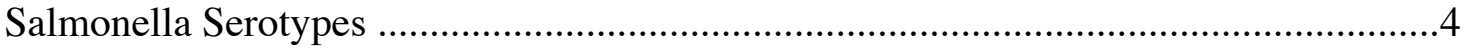

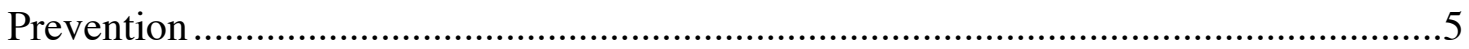

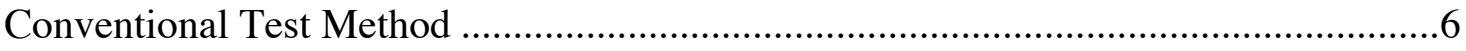

Paper-based analytical devices ( $\mu$ PADs) .......................................................

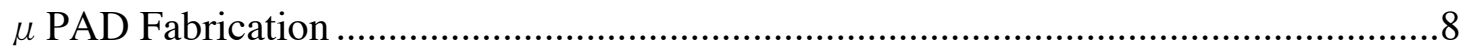

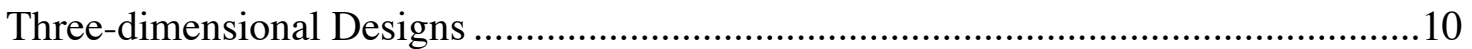

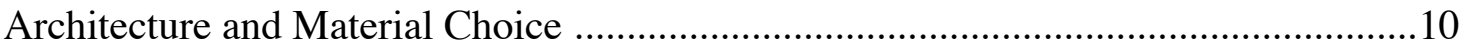

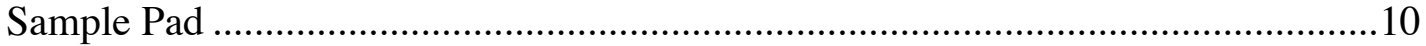

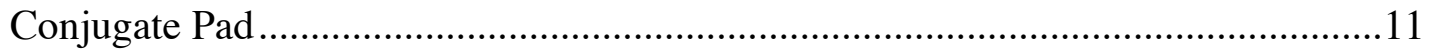

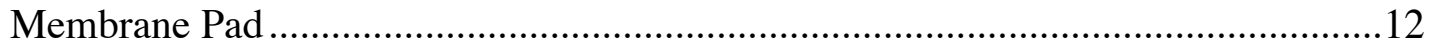

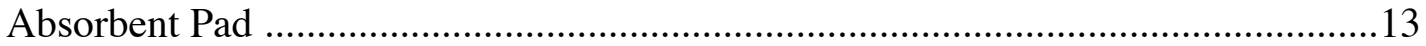

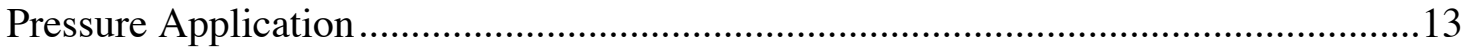


Immunoassay

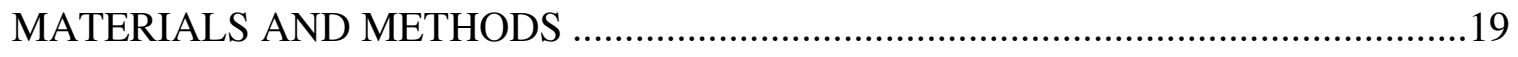

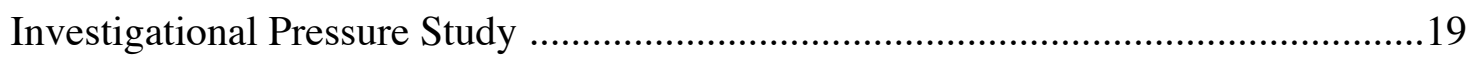

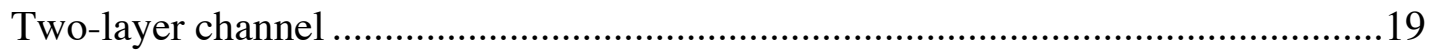

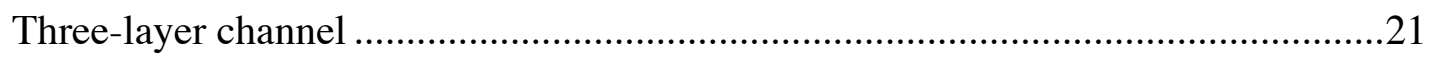

Simple pressure housing design .....................................................................22

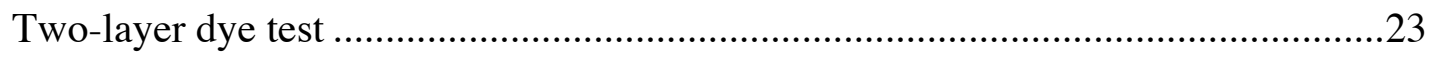

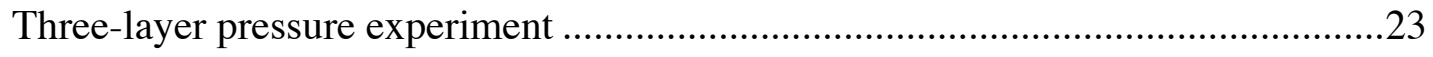

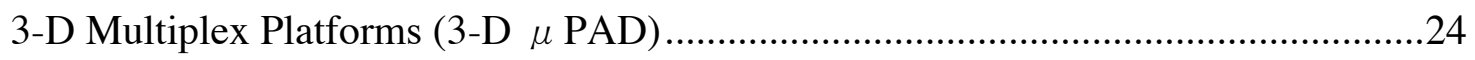

Multiplex Channel Design 1 .........................................................................24

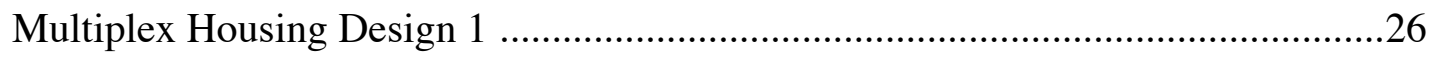

Multiplex Channel Design 2 .................................................................................2

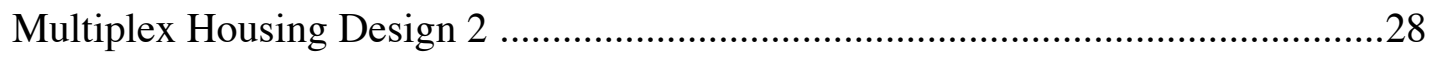

Multiplex Channel Design 3 ................................................................................29

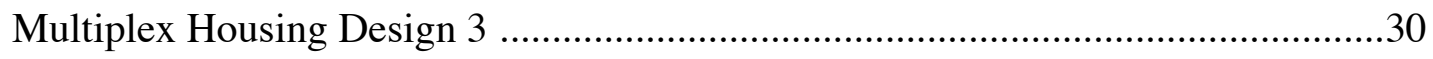

Multiplex Channel Design 4 ..................................................................................

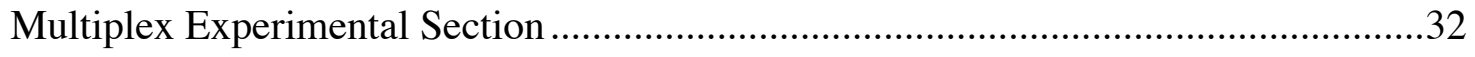

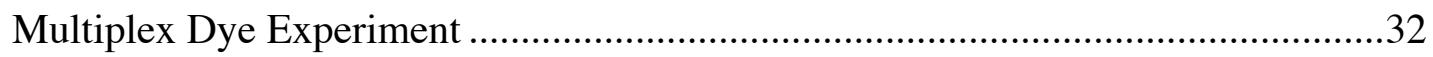

Multiplex Glucose Experiment .............................................................................32

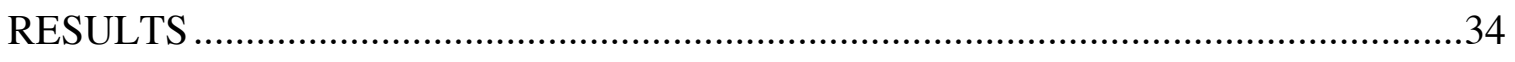

Investigational Pressure Testing .....................................................................................

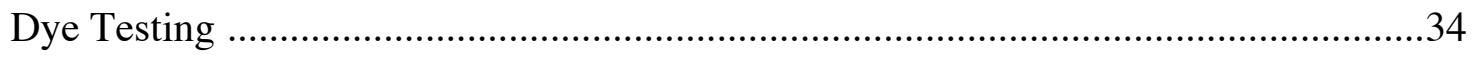

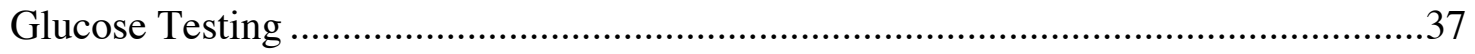




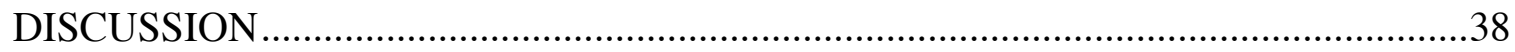

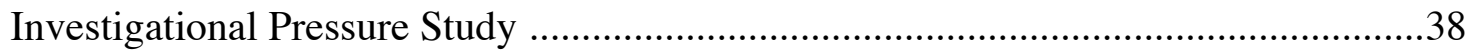

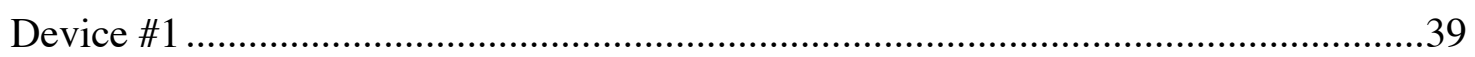

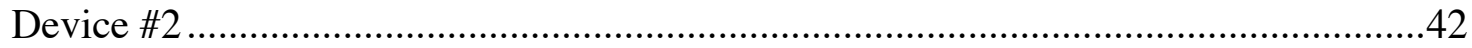

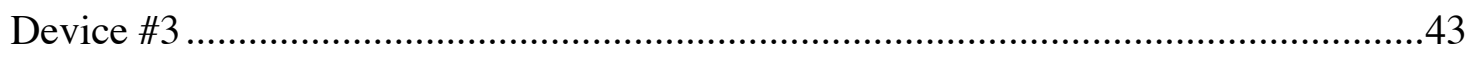

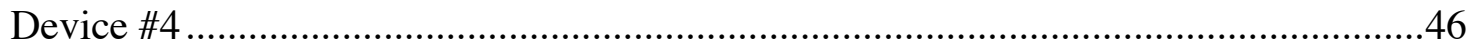

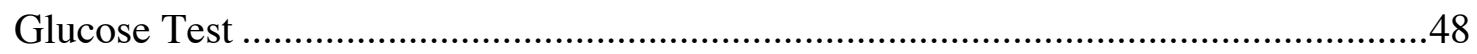

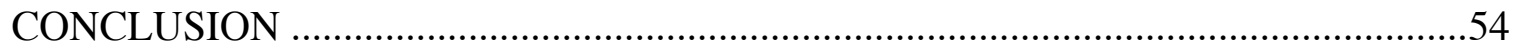

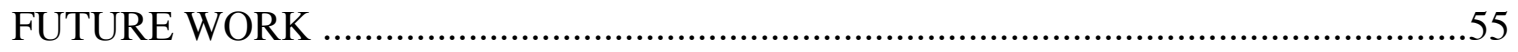

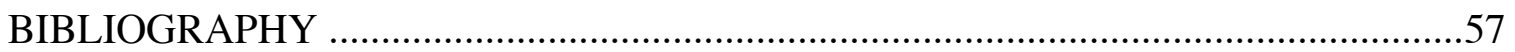




\section{LIST OF TABLES}

Table

Page

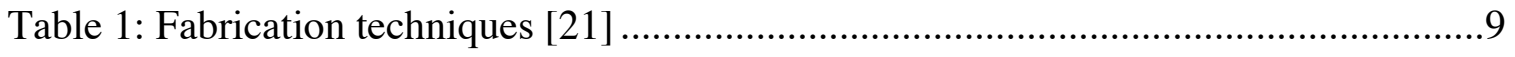

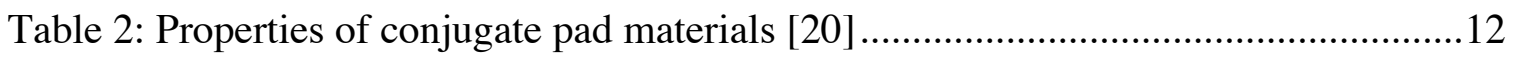

Table 3: Binding properties of different membrane polymers [20] ..............................12

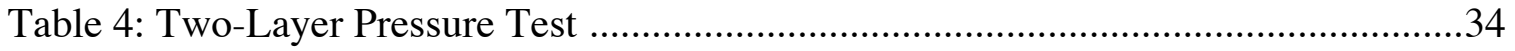

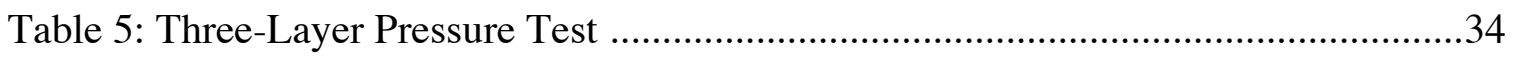

Table 6: Device \#1 Dye Test Development Times ...................................................34

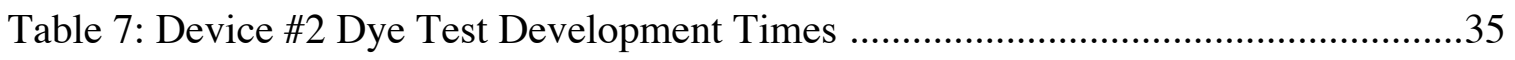

Table 8: Device \#3 Dye Test Development Times ....................................................35

Table 9: Device \#4 Dye Test Development Times ....................................................36

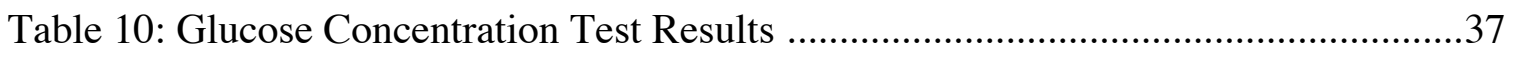




\section{LIST OF FIGURES}

Figure

Page

Figure 1: Incidence of Salmonella infections per 100,000 persons in the

United States - FoodNet, 1996 - 2014 [6]

Figure 2: Percentage of Salmonella infections by serotype - FoodNet, $2014[6]$

Figure 3: Basic Outline of The Selective Culture Method [11] ......................................6

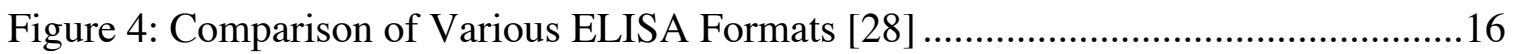

Figure 5: Schema of the aptamer based detection. (A) A schematic

description of the magnetic enrichment of bacteria and SDA amplification of aptamer. Bacteria are first mixed with excess amount of aptamers. After incubation, streptavidin coated magnetic beads are added, and the MNBAp-bacteria complexes are collected by a magnet separator. The collected MNB-Ab-bacteria complexes are directly amplified by isothermal strand

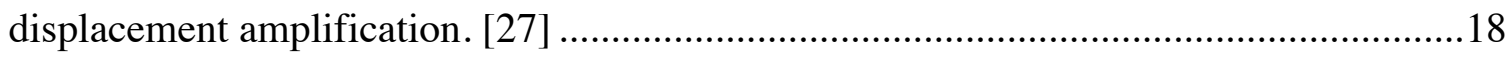

Figure 6: AutoCAD Two-Layer Channel Design ....................................................20

Figure 7: Xerox Color Cube 8570 Wax Printer...........................................................20

Figure 8: Clarkson Digital Ceramic Top Hot Plate ..................................................2 21

Figure 9: AutoCAD Three-layer Channel Design .......................................................22

Figure 10: Investigational Pressure Study AutoCAD Housing Design ...........................22

Figure 11: Fluid Routing Layer AutoCAD Design (Left) and Isolation

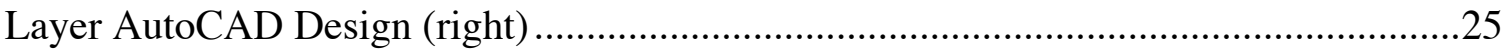


Figure 12: AutoCAD Design of Conjugate Layer (left) and the Conjugate

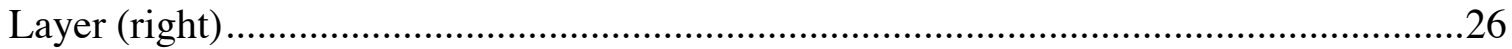

Figure 13: Multiplex Housing Design \#1 AutoCAD Drawing .....................................27

Figure 14: AutoCAD Drawing of Modified Isolation Layer........................................27

Figure 15: AutoCAD Drawing of Conjugate Layer (left), Detection Layer

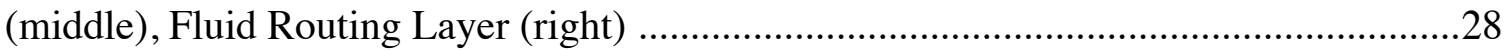

Figure 16: Multiplex Housing \#2 AutoCAD Drawing. Bottom section

(left), middle section (center), top section (right)

Figure 17: AutoCAD Drawing of Channel Network Design \#3 Top:

Barrier Layer, Fluid Routing Layer. Bottom: Conjugate Layer, Detection

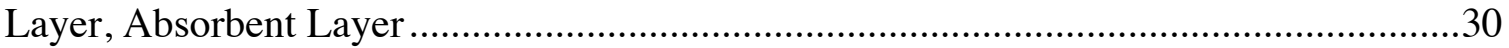

Figure 18: Multiplex Housing Design \#3 AutoCAD Drawing ...................................... 31

Figure 19: Multiplex Channel Network Design \#4. Top: Barrier Layer,

Fluid Routing Layer. Bottom: Conjugate Layer, Detection Layer,

Absorbent Layer

Figure 20: Mean channel development time from dye testing of device \#4.

Development times are reported in seconds. (* Indicates a significant

difference in the mean channel development time between channel 5 and

channels 1 and $3(\mathrm{p}<0.05)$.)

Figure 21: Top view of the fluid routing layer from the first dye test of device \#1 showing the lack of overlap between the conjugate layer and the fluid routing layer. 40 
Figure 22: Top view of the fluid routing layer from dye test showing the misalignment of a layer in channel 2 .

Figure 23: Results from the fourth dye test. Channel 2 was observed to have incomplete fluid routing and channel 4 had unsuccessful fluid routing .44

Figure 24: Dye test results from the third test (left) and fifth test (right). .45

Figure 25: Result from the sixth dye test. Also shown are the three alignment holes and the rubber tubing.

Figure 26: Result from the $1.2 \mathrm{mM}$ glucose concentration testing.

Blue/green color indicates the presence of glucose

Figure 27: Reagents deposited via a reagent pencil in low (left) and high

(right) quantities in the conjugate zones.....

Figure 28: The leading edge of the fluid during the glucose concentration

test.

Figure 29: Signal intensity from the $1.2 \mathrm{mM}$ glucose concentration test after 5 minutes.

Figure 30: Signal intensity from the $1.2 \mathrm{mM}$ glucose concentration test after 5 minutes. 


\section{INTRODUCTION}

\section{Thesis Specific Goals}

Paper-based microfluidic devices, also known as microPADs, are small and portable devices made out of patterned paper that offer a promising platform for diagnostic applications outside of a laboratory. These devices are easy to use, low-cost, and capable of functioning in almost any environment. MicroPADs ( $\mu$ PADs) function without supporting equipment like pumps and require only microliter volumes of sample and microgram quantities of reagents. MicroPADs do not require sophisticated equipment to read the results and most can seen by the naked eye. For theses reasons and many others, microPADs are an attractive option in diagnostic applications.

The primary goal of this thesis was to develop a device capable of multi-target detection. In order to guide this work a number of specific goals were determined:

\#1: Can a paper-based analytical device ( $\mu$ PAD) be designed for simultaneous detection of 5 targets?

\#2: Will the use of rubber cord stock to apply pressure along the edge of the channels allow for successful fluid conductivity between layers?

\#3: Can the device successfully incorporate the use of the reagent pen for the detection of glucose? 
The methods and results in an attempt to meet these goals are discussed in detail in the remained of this report.

\section{Overview}

Over the last 100 years, Salmonella bacteria have been identified as the most common primary cause of foodborne illness in many countries, including the United States. [1] [2] [3] Despite increase in food safety regulations and strict adherence to good manufacturing practices (GMP) [4] and good agricultural practices (GAP) [5], the incidence and severity of human salmonellosis continues to remain a serious issue. In the United States, the Foodborne Diseases Active Surveillance Network (FoodNet) has been responsible for monitoring the incidence of Salmonella linked infections since its creation in 1996. Annual reports from FoodNet indicate that the incidence of Salmonella infections has increased from rate in 1996 (Fig. 1).

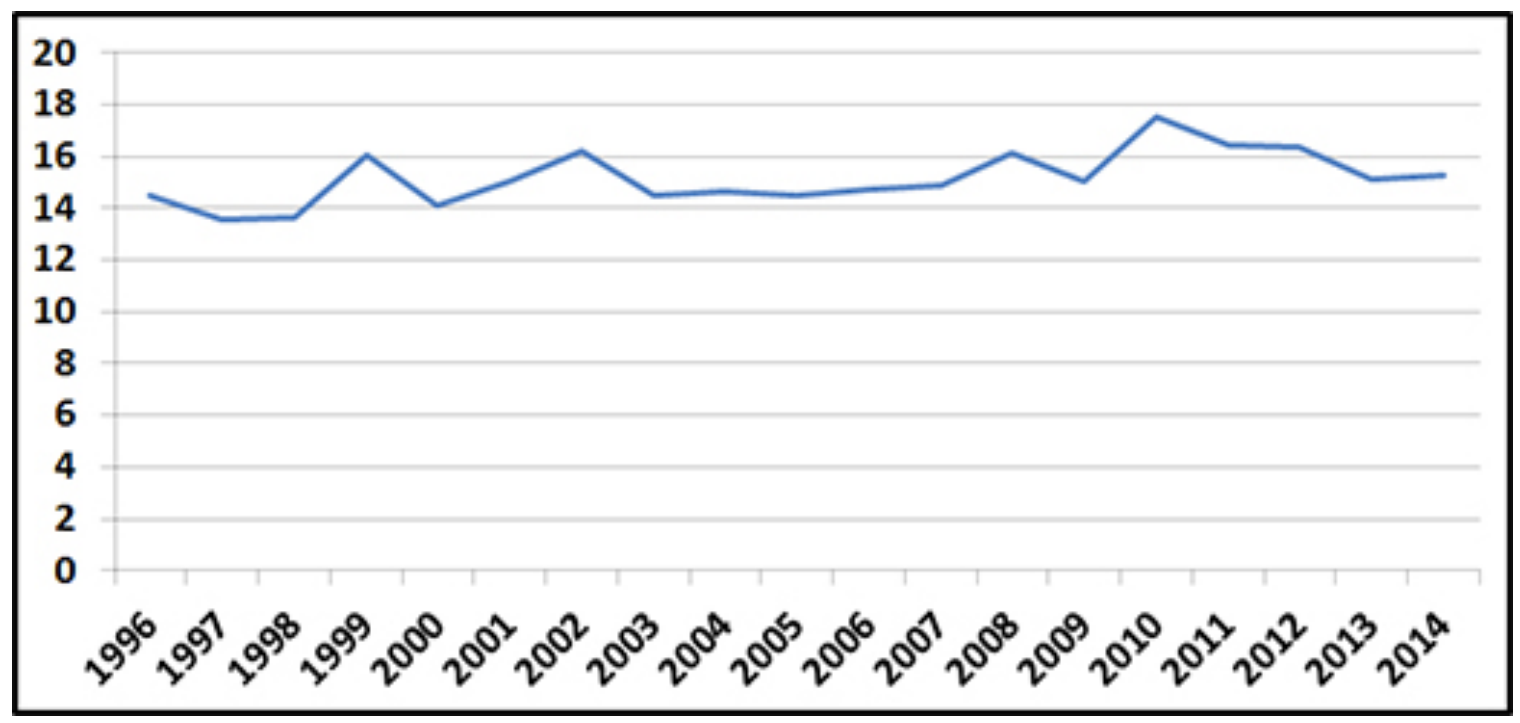

Figure 1: Incidence of Salmonella infections per 100,000 persons in the United States - FoodNet, 1996 $-2014[6]$ 
The Centers for Disease Control and Prevention (CDC) estimates that approximately 1.2 million human Salmonella infections occur annually in the United States, resulting in 19,000 hospitalization with nearly 450 deaths [6]. The estimated total cost associated with Salmonella infections may exceed 1 billion dollars annually. [7]

\section{The Disease}

Salmonellosis is a disease caused by the bacteria Salmonella. It is usually characterized by the acute onset of fever, abdominal pain, diarrhea, nausea and sometimes vomiting [8]. The onset of disease symptoms occurs 6 - 72 hours (usually 1236 hours) after ingestion of salmonella, and illness lasts 2-7 days. In most cases the symptoms are relatively mild and patients will recover without specialized treatment [8]. However, in patients who are very young or are elderly, the associated dehydration can become severe and life threatening. Although large Salmonella outbreaks usually attract media attention, 60 to $80 \%$ of all salmonellosis cases are not recognized as part of a known outbreak and are classified as sporadic cases, or are not diagnosed as such at all [8].

\section{Sources and Transmission}

Salmonella bacteria are widely distributed in domestic and wild animals. They are prevalent in food animals such as poultry, pigs, cattle; and in pets, including cats and dogs, birds and reptiles such as turtles [8]. Salmonella can pass through the entire food chain from animal feed, primary production, and all the way to households or food-service establishments and institutions. Salmonellosis in humans is generally contracted through the consumption of contaminated food of animal origin (mainly 
eggs, meat, poultry and milk), although other foods, including green vegetables

contaminated by manure, have been implicated in its transmission. [8]

\section{Salmonella Serotypes}

Currently, over 2500 serotypes have been identified for Salmonella. However, it is estimated that less than 100 serotypes account for most human infections with the five most common comprising the majority of cases. Since 2010, FoodNet has reported that the five most common serotypes linked to human illness have been Enteritidis, Typhimurium, Newport, Javiana, and I 4,[5],12,i- .

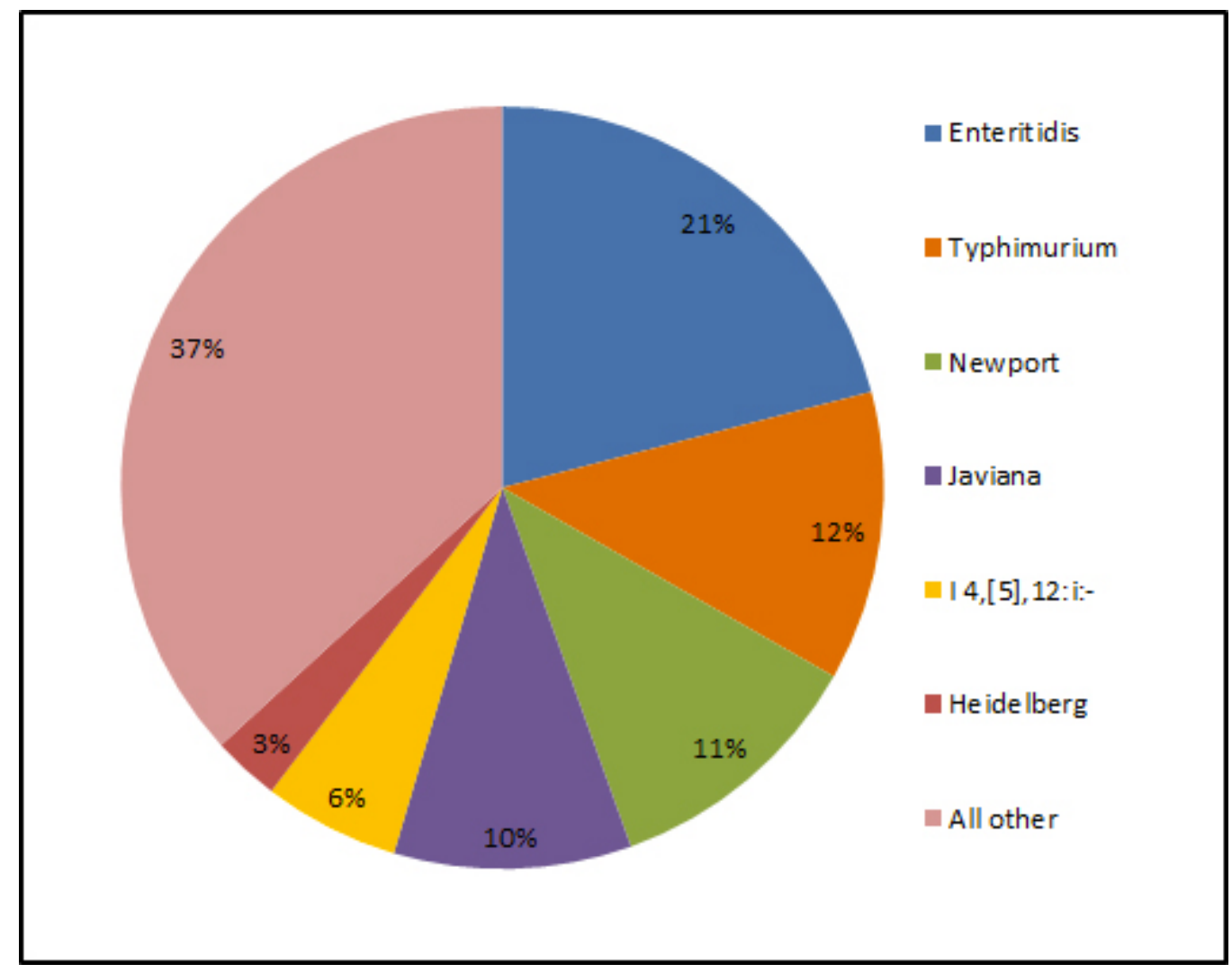

Figure 2: Percentage of Salmonella infections by serotype - FoodNet, 2014 [6] 
In the FoodNet 2014 Annual Foodborne Illness Surveillance Report, Salmonella Enteritidis was the most commonly reported serotype in causing human illness and was responsible for $21 \%$ of cases. Enteritidis is most commonly associated with poultry however; outbreaks of this serotype have also been associated with shell eggs, alfalfa sprouts, pine nuts, and ground beef. [9] Salmonella Typhimurium was the second most common serotype with $12 \%$ of cases. Typhimurium is most often identified in bovine products although it has also been linked to pork and other poultry products. Salmonella Newport was the third most common serotype accounting for $11 \%$ of cases. This strain is most often associated with turkey products but outbreaks have been linked to cantaloupe, live poultry, and alfalfa sprouts. Salmonella Javiana was the fourth most common serotype with $10 \%$ of cases. Reports from the U.S. Department of Agriculture, indicate that this strain is not often associated with products that are subject to industry regulation. Javiana is typically associated with exposure to amphibians in the Southeast U.S but has also been linked to contaminated mozzarella cheese, watermelon, bass, poultry, lettuce and tomatoes. [10] Salmonella I 4,[5],12,i- was identified as the fifth most common serotype with $6 \%$ of cases. This strain has been linked to chicken meat and has also been found in shell eggs.

\section{Prevention}

In order to improve food safety, Salmonella surveillance and monitoring should be based on reliable and efficient detection methods [11]. It is essential that surveillance and monitoring should cover the entire food chain, preferably starting from investigation of feed and feed ingredients for Salmonella contamination [11]. Ideal detection methods should be low cost, easy to use, and produce results in a short period of time. 


\section{Conventional Test Method}

The conventional test for Salmonella is a selective culture method. This

involves a nonselective pre-enrichment of a defined weight or volume of the sample,

followed by a selective enrichment step, plating onto selective agars, and biochemical and serological confirmation of suspect colonies [11].

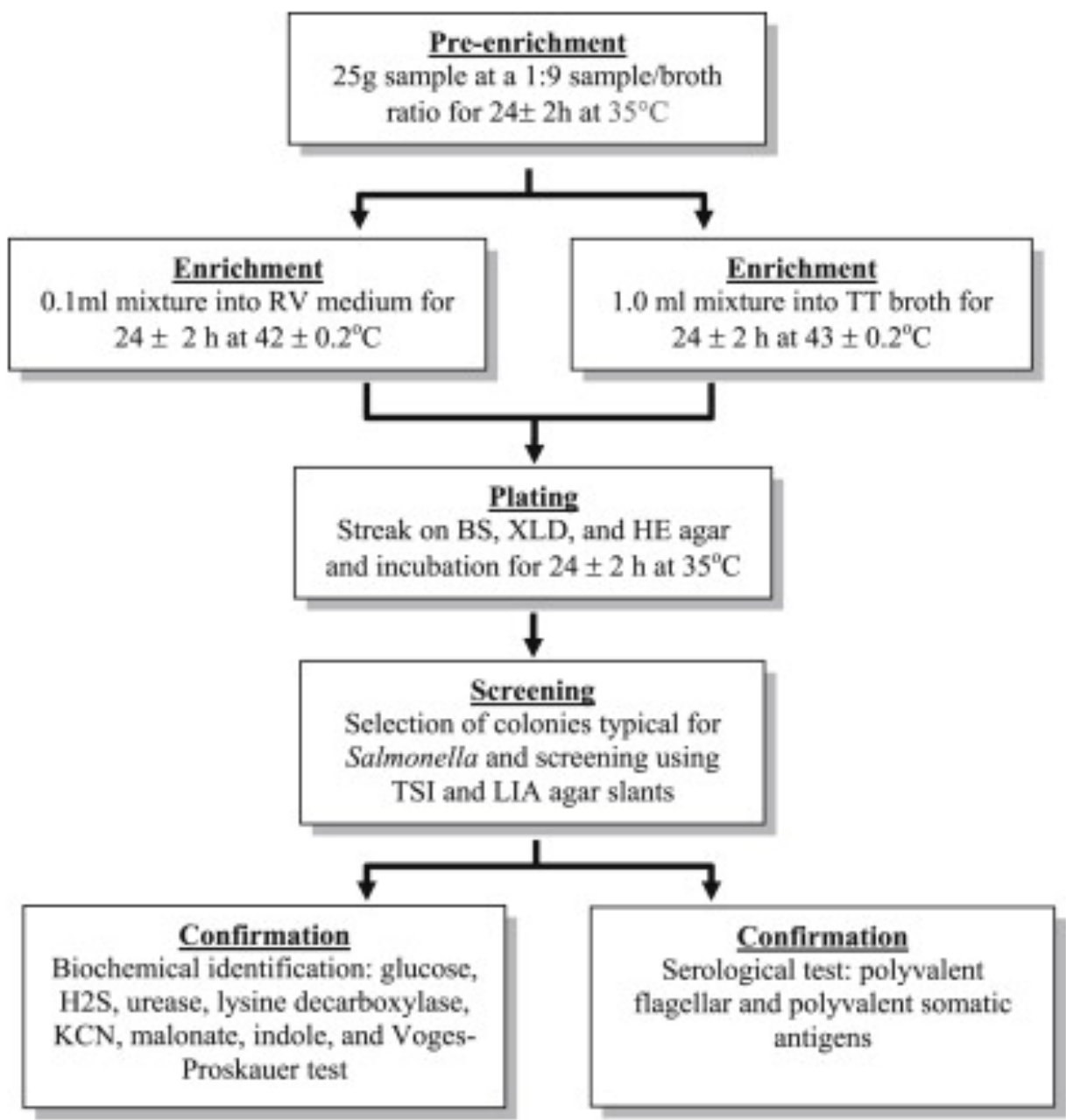

Figure 3: Basic Outline of The Selective Culture Method [11] 
This method serves as the basis for analysis in many food safety and public health laboratories due to the ease of use, reliability of results, high sensitivity and specificity, and lower cost compared to emerging molecular-based technologies [12] [13]. However, this method is not without its drawbacks. This method requires not only a laboratory with specialized equipment, but highly trained personnel and an extended period of time. A typical procedure requires the preparation of multiple subcultures, which can take more than 5 days for complete isolation and confirmation. In addition, false positive results may occur due to competitive flora [14] [15]. Even with improvements to the current method, for example the use of chromogenic and fluorogenic growth media, the test results using these selective media is typically available only 1 day earlier and are not fast enough to respond to Salmonella outbreaks and product recalls [1] [13] [16].

One proposed alternative to the conventional culture method is a lateral flow assay. A lateral flow assay is a simple platform capable of testing for a specific analyte in a solution. Theses test are very quick and depending on the target, can have great sensitivity. The one drawback though, is that typically only a single analyte can be detected. This can be an issue when testing for Salmonella since multiple strains can be found from the same type of food source. It would therefore be beneficial to be able to have a custom lateral flow assay that would be capable of detecting multiple targets in a single test. In fact, custom lateral flow assays have been the source of extensive research over the years and have become known as microPADs ( $\mu$ PADs).

\section{Paper-based analytical devices ( $\mu$ PADs)}

In the past few years, paper-based analytical devices ( $\mu$ PADs) have become attractive alternatives to conventional methods as an inexpensive assay platform [20]. 
Paper is ideal for fabricating low cost microanalytical devices for a number of reasons: 1) paper is available everywhere and is inexpensive $\left(\sim \$ 6 / \mathrm{m}^{2}\right.$ for high-quality chromatography paper). Simple $\mu$ PADs can be fabricated for only a few cents; 2) paper wicks aqueous fluids and provides passive transport without any additional equipment; 3 ) paper has been used for decades and can leverage existing analytical techniques [21]. Additional advantages of $\mu$ PADs also include small (microliter volumes and nanogram masses) sample and reagent consumption, simple operation and manufacturing, portability, disposability, an extensive application base, a high surface area relative to traditional microfluidics for analyte capture and visualization and potential for use in scenarios where minimal instrumentation is required [17] [21].

\section{MPAD Fabrication}

With improvements in $\mu \mathrm{PAD}$ technology, a number of fabrication techniques have been established. Currently, fabrication techniques include: photolithography, inkjet printing, stamping, cutting, screen-printing, and wax printing [21]. 
Table 1: Fabrication techniques [21]

\begin{tabular}{|l|l|l|}
\hline Method & Advantages & Disadvantages \\
\hline Photolithography & Can pattern a wide variety of papers & $\begin{array}{l}\text { Hydrophilic areas exposed to polymers and } \\
\text { solvents }\end{array}$ \\
\hline Plotting & $\begin{array}{l}\text { Hydrophilic channels not exposed to } \\
\text { polymers }\end{array}$ & Requires a customized plotter \\
\hline Inkjet etching & $\begin{array}{l}\text { Reagents can be inkjet printed into the } \\
\text { test zones using the printer }\end{array}$ & $\begin{array}{l}\text { Requires a customized inkjet printer; } \\
\text { hydrophilic areas exposed to polymers and } \\
\text { solvents }\end{array}$ \\
\hline Plasma etching & $\begin{array}{l}\text { Useful for laboratories equipped with a } \\
\text { plasma cleaner that wish to make many } \\
\text { replicates of a few simple patterns }\end{array}$ & $\begin{array}{l}\text { Hydrophilic areas exposed to polymers and } \\
\text { solvents, metal masks must be made for } \\
\text { each pattern; cannot produce arrays of free- } \\
\text { standing hydrophobic patterns }\end{array}$ \\
\hline Cutting & $\begin{array}{l}\text { Hydrophilic channels not exposed to } \\
\text { polymers or solvents }\end{array}$ & $\begin{array}{l}\text { Devices must be encased in tape; cannot } \\
\text { produce arrays of free-standing hydrophilic } \\
\text { patterns }\end{array}$ \\
\hline Wax printing & $\begin{array}{l}\text { Rapid }(\sim 5 \text { minutes); requires only a } \\
\text { commercially available printer and hot } \\
\text { plate; hydrophilic channels not exposed } \\
\text { to polymers or solvents }\end{array}$ & $\begin{array}{l}\text { The design of the patterns must account for } \\
\text { the spreading of the wax in the paper }\end{array}$ \\
\hline
\end{tabular}

While each of these methods has its own advantages and disadvantages, it is

thought that wax printing is the fastest, simplest fabrication method to date. A wax printer employs melted wax in the same way an ink jet printer uses ink cartridges. The solid wax patterns are first printed onto the top of the paper. The wax in then re-melted and a hydrophobic barrier is created as the wax penetrates the paper fully. The barriers created then enable spatial control over fluid transport caused by the capillary flow in the paper. To date, $\mu$ PADs generally have two fundamental design schemes: spot tests and fluidic networks [17]. The first design type, the spot test, is a simple design in which the sample and reagents remain stationary within the device [22]. The second design, describes a fluidic network on paper where sample and reagents are transported throughout the device via capillary action. In recent years, fluidic networks have evolved 
from simple lateral flow assays to more complex, three-dimensional designs [23]. The design in used in this thesis is a three-dimensional fluidic network.

\section{Three-dimensional Designs}

3D paper-based systems are attractive diagnostic platforms because of the ability to combine, at a low cost, simplicity in fabrication and complexity in fluidic and bioanalytical capability [23]. They have the ability to transport fluid both vertically and laterally, and can enable streams of fluid to cross one another without mixing [23]. These devices take advantage of capillary action to distribute fluids into complex arrays of tens to thousands of detection zones at different times depending on the architecture of the device and choice of materials [23]. These devices are also small, lightweight, and are each to stack, store and transport. Since they do not require external pumps to function they are suitable for use in environments outside of a lab.

\section{Architecture and Material Choice}

Three-dimensional $\mu$ PADs are typically comprised of at least four unique types of layers: sample pad, conjugate pad, membrane pad, and absorbent pad. Fluid that is transported through the device will usually encounter the sample pad first, and then proceed through the conjugate, membrane, and lastly the absorbent pad.

\section{Sample Pad}

The sample pad is typically made from cellulose and/or glass fiber [24]. The main function of the sample pad is to conduct fluid to the other components of the device. It is necessary that the sample pad conduct fluid in a smooth, continuous, and homogenous manner to ensure accurate results at the end of the test [24]. In some assays the sample 
pad may also be designed to pretreat the sample before it's conducted to the next component. Pretreatment could include separation of sample components, removal of interfaces, adjustment of $\mathrm{pH}$, etc., depending on how the device is intended to function [24].

\section{Conjugate Pad}

The conjugate pad holds the dried reagents. Since the test sample will be continuously moving through the pad, the material of the conjugate pad should immediately release labeled conjugate upon contact the sample. The materials commonly used for conjugate pads are non-woven fibers, which are manufactured by compressing pre-spun fibers of cellulose, glass, or plastic into thin mats [25]. When the test sample flows into the conjugate pad, the detector reagent solubilizes, lifts off the pad material, and moves with the fluid front. An important function of the conjugate pad is uniform transfer of the detector reagent and test sample to the membrane layer. Ultimately, the sample volume required to release the detector particle into the sample stream determines how much analyte can be measured [25]. This is one of the more important design considerations as the sample volume actually analyzed in the test equals the amount of sample required to solubilize the detector particles, not the total amount absorbed by the device. 
Table 2: Properties of conjugate pad materials [20]

\begin{tabular}{|l|l|l|l|}
\hline Non-woven Material & Brief Description & Key Benefits & Key Liabilities \\
\hline Glass fibers & $\begin{array}{l}100-500 \mu \mathrm{m} \text { thick, may } \\
\text { contain binders to hold } \\
\text { fibers together }\end{array}$ & $\begin{array}{l}\text { Good hold-up volumes, } \\
\text { low nonspecific binding }\end{array}$ & $\begin{array}{l}\text { Poor tensile properties, } \\
\text { difficult to slit and web } \\
\text { handle }\end{array}$ \\
\hline Cellulose Filters & $\begin{array}{l}300-100 \mu \mathrm{m} \text { thick, } \\
\text { compact fibers of } \\
\text { consistent density }\end{array}$ & $\begin{array}{l}\text { Very low nonspecific } \\
\text { binding, normally very } \\
\text { uniform }\end{array}$ & $\begin{array}{l}\text { High hold-up volumes } \\
\left(>50 \mu \mathrm{l} / \mathrm{cm}^{2}\right), \text { can be } \\
\text { very weak when wet }\end{array}$ \\
\hline $\begin{array}{l}\text { Surface-modified } \\
\text { polyester }\end{array}$ & $\begin{array}{l}100-300 \mu \mathrm{m} \text { thick, } \\
\text { hyropphilic polyester } \\
\text { fibers }\end{array}$ & $\begin{array}{l}\text { Low nonspecific } \\
\text { binding, excellent } \\
\text { tensile strength and web } \\
\text { handling }\end{array}$ & $\begin{array}{l}\text { Low, and somewhat } \\
\text { variable, hold-up } \\
\text { volumes }\left(<15 \mu \mathrm{l} / \mathrm{cm}^{2}\right)\end{array}$ \\
\hline
\end{tabular}

\section{Membrane Pad}

The membrane pad is probably the single most important material used in a $\mu \mathrm{PAD}$. The physical and chemical attributes of the membrane affect its capillary flow properties [25]. The capillary flow properties in turn affect reagent deposition, assay sensitivity, assay specificity, and test line consistency [25]. For device to work, the membrane pad must irreversibly bind capture reagents in the test and control zones. The polymer from which the membrane is made determines most of its binding characteristics.

Table 3: Binding properties of different membrane polymers [20]

\begin{tabular}{|l|l|}
\hline Membrane Polymer & Primary Binding Mechanism \\
\hline Nitrocellulose & Electrostatic \\
\hline Polyvinylidene fluoride & Hydrophobic (Charge-modified) \\
\hline Nylon & (Ionic) electrostatic \\
\hline Polyethersulfone & Hydrophobic \\
\hline
\end{tabular}

Nitrocellulose is commonly used as a membrane material as these membranes are easy to use, inexpensive, and offer high affinity for proteins and other biomolecules [24]. 


\section{Absorbent Pad}

The primary function of the absorbent pad is to increase the total volume of sample that can be transported through the $\mu \mathrm{PAD}$. It plays a role in maintaining the flow rate of the liquid in the previous layers and stops back flow of the test sample. The increased volume can be also used to wash unbound detector particles away from test and control lines, thereby lowering the background and enhancing the assay sensitivity [24]. Typically, most absorbent pads are made from cellulose filters. However, since the absorbent pad has fewer physical and chemical requirements, other materials may also be used.

\section{Pressure Application}

An important design consideration in a $\mu \mathrm{PAD}$ is how to achieve successful fluid routing from one layer to another. It should be noted that successful fluid routing in this context is defined as the smooth, continuous, and homogenous flow of fluid between two different layers. While capillary action is sufficient to allow for fluid routing within the same layer, it is not a strong enough force to promote successful fluid routing between different layers that are simply lying on top of each other. Therefore alternative methods need to be incorporated into the $\mu \mathrm{PAD}$ design to promote fluid flow between layers. Historically this has been accomplished with an external housing unit however, recent research has developed an alternative method that does not require an external housing unit.

The traditional method for promoting fluid flow between layers has been to incorporate an external housing unit that would apply pressure through the use of strategically placed tabs at the transitional zones between layers. The tabs were designed 
such that the pressure would be applied orthogonal to the channel at the site where two different layers overlap. One concern with this method is that the pressure is applied directly across the channels. Since the membranes used are relatively weak structurally, they are not capable of resisting large compressive loads. If the pressure applied from the tab is too great it can cause the membrane pores to collapse. The pores may collapse slightly which could hinder or block larger molecules from flowing through the membrane resulting in an invalid test; or the pores may collapse completely ceasing all fluid flow. A secondary concern are that tabs the may come into direct contact with the fluid in the membrane and may have an unintended consequence.

Recently, Martinez et al. developed a novel method for fabricating $\mu$ PADs without the use of an external housing unit [23]. The $\mu$ PAD they developed was primarily held together using double-sided tape. Although the tape was successful in keeping the layers together, it introduced a gap between the layers. Therefore, to bridge the gap a cellulose paste was added to the device. As a result, successful fluid routing was achieved in the study by Martinez et al. [23]. However, in another study performed by Jennifer Ward, this method was described this method as "not always the most efficient and reliable" [26]. It was also suggested that the assembly process using these coupling agents was difficult, time consuming, and would be hard to scale up for mass production [26]. Since alternative methods for promoting fluid flow between layers are lacking in functionality, it is still necessary to incorporate an external housing unit into the $\mu$ PAD design.

Previous work by Mackenzie Tageson, involved the use of external housing units with pressure tabs that were laser cut from acrylic [27]. The final design had four 
pressure tabs that directly covered the four channels from the conjugate pad to the end of the channel. Successful fluid routing was achieved in all four channels however; it was observed that the development times for each channel were found to be statistically different [27]. Additionally, previous experiments also observed that the tabs completely restricted fluid flow when the bolts of the housing unit were tightened too much [27]. Also present with this design is the concern that the direct contact between the tab and fluid may alter the performance of the test.

Therefore, in order to address the pressure design requirement and also address the concerns previously mentioned, the work in this thesis presents a new method of applying pressure.

\section{Immunoassay}

The final component of a fully functional $\mu \mathrm{PAD}$ is the detection method. Ideally, a $\mu$ PAD should incorporate a detection method that is simple, sensitive, highly selective, and cost-effective [26]. Immunoassay, a procedure for detecting or measuring specific proteins, is considered as one of the most widely used techniques for protein detection due to its high sensitivity, high specificity, excellent accuracy, and practicality [26]. To date, various methods and strategies based on different signal-transducer principles have been reported and developed for immunoassays, e.g. surface plasmon resonance, fluorescence (FL), chemiluminescence (CL), and colorimetric method [26]. Typically, the colorimetric method is the most commonly used method in immunoassays because of several important advantages: simplicity, practicality, low cost, and rapid/direct readout with the naked eye [26]. 
Among the known colorimetric techniques, enzyme-linked immunosorbent assay (ELISA) has been widely used for analysis due to its ease of use and ability to handle a large number of samples. An ELISA is usually comprised of a capture reagent, detection reagent, and a signal indicator. Depending on the format used, e.g. direct, indirect, sandwich, or competitive, the reagents will interact in different ways.

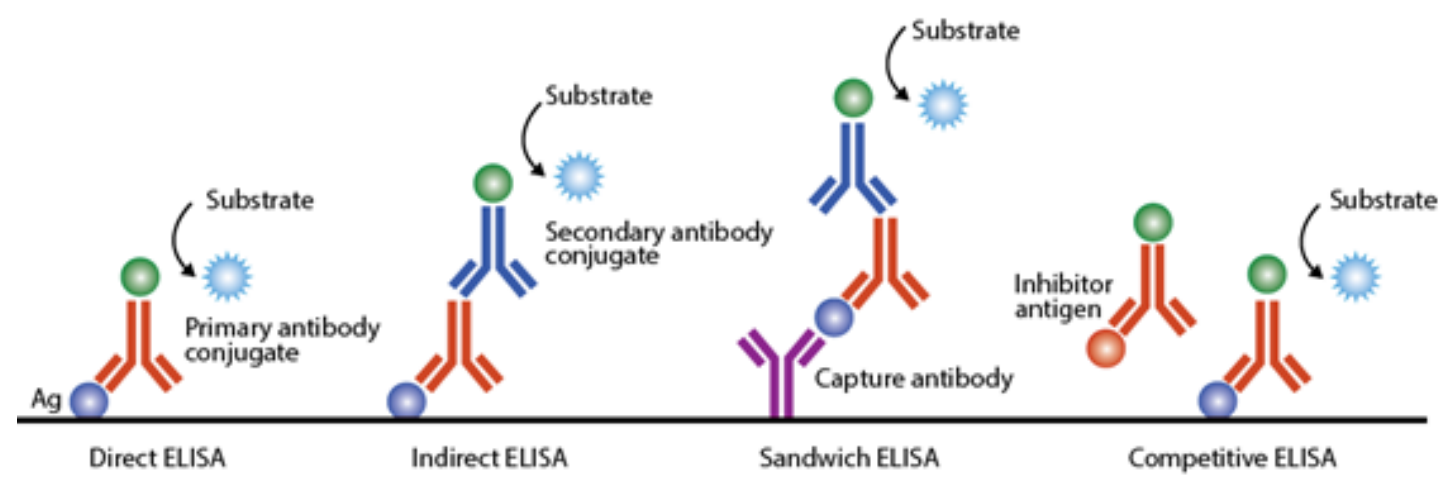

Figure 4: Comparison of Various ELISA Formats [28]

Additionally, ELISA is compatible with a number of signal indicators. To date, several labels have been proposed to serve as signal indicators in paper-based assays although colloidal gold nanoparticles (Au-NPs) are the common choice [27]. Colloidal gold nanoparticles (Au-NPs) are typically used as a signal indicator because of their ease of preparation, capability to adsorb proteins, and detectability to the naked eye [28].

Despite the numerous benefits a standard ELISA offers, very few studies have achieved an acceptable level of sensitivity for the detection of foodborne Salmonella [29]. Ideally it is desirable to be able to detect a single bacteria cell in a sample however, detection limits of less $10^{2} \mathrm{CFU} / \mathrm{ml}$ are generally considered acceptable [29]. Current methods typically have a detection level of around $10^{4} \mathrm{CFU} / \mathrm{ml}$ [29]. For the most part, the detector reagent is what determines the sensitivity of the assay. The detector reagents 
used in a standard ELISA are either an antigen or antibody. While, antigens and antibodies are used because they do offer good selectivity for targets its only under ideal conditions that they achieve an acceptable level of sensitivity. Recent research suggests that aptamers may offer improvement over the traditional detector reagents, specifically in the detection of foodborne Salmonella [27].

Aptamers are single stranded oligonucleotides that have high affinity to certain targets and are widely used for analyte detection. Moreover, aptamers are inexpensive, stable, and can be chemically synthesized in high purity and modified with relative ease [27]. In 2010, Fang et al. developed an aptamer-based biosensor for simple and rapid detection of viable Salmonella [27]. Two aptamers against different outer membrane proteins were used in the detection. Biotin modified capture-aptamer (c-aptamer) was used for the bacteria enrichment. Another unmodified amplification-aptamer (a-aptamer) against another outer membrane protein was used as template for strand displacement amplification (SDA) [27]. The amplified single strand DNA was then detected with a lateral flow assay. Figure 5 illustrates the process behind the aptamer-based biosensor. 


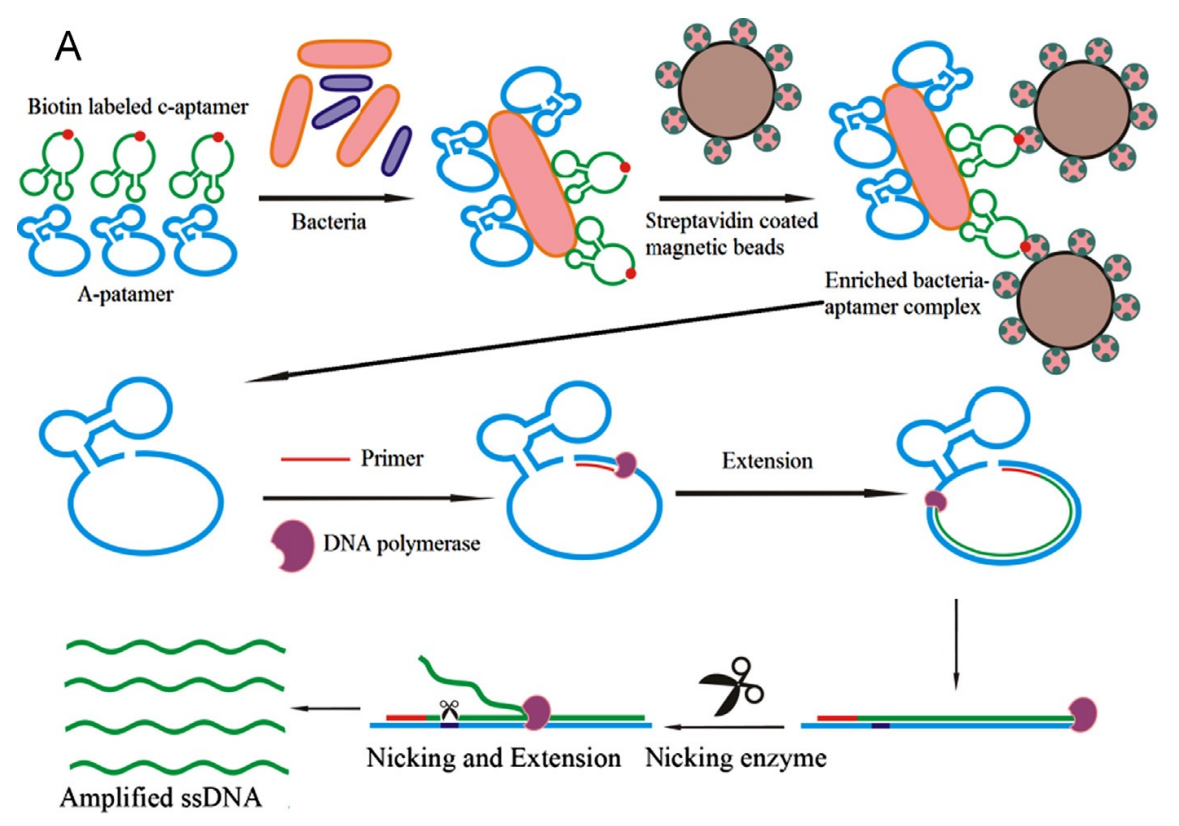

Figure 5: Schema of the aptamer based detection. (A) A schematic description of the magnetic enrichment of bacteria and SDA amplification of aptamer. Bacteria are first mixed with excess amount of aptamers. After incubation, streptavidin coated magnetic beads are added, and the MNBAp-bacteria complexes are collected by a magnet separator. The collected MNB-Ab-bacteria complexes are directly amplified by isothermal strand displacement amplification. [27]

The aim of this thesis is to document the creation a custom $\mu$ PAD that will allow for the simultaneous detection of multiple targets. In the Material and Methods section, the device designs of the channel networks and housing units are discussed, along with the device testing protocols. The results from the fluid conductivity tests and the glucose reagent pencil tests are then reported and discussed. A conclusion of the work is presented followed by possible future work. 


\section{MATERIALS AND METHODS}

In order to address the specific aims set forth at the start of this thesis, numerous devices and experimental tests were developed. The information in this section explains the design process, fabrication, and experimental methods used to validate the performance of newly created $\mu$ PADs. This section begins with the methods behind the initial investigatory work for the new pressure application. Then talks about design and testing of the multiplex $\mu$ PADs. Lastly includes testing with dye and the first time use of a reagent pen for glucose testing.

\section{Investigational Pressure Study}

The following subsections describe the methods used for the investigational work into the new pressure application. Specifically these subsections consist of two channel designs, an accompanying housing design, and lastly experimental testing.

\section{$\underline{\text { Two-layer channel }}$}

The first channel design of the pressure study was comprised of two fluid routing layers. The bottom layer consisted of a sample input zone attached to a straight rectangular channel that extended approximately half the length of the layer. The top layer consisted of an identical rectangular channel that overlapped part of the first layer and continued to the end of the top layer. The widths of the channels were designed to be $2.5 \mathrm{~mm}$ wide and the overlap of the two layers was designed to be $2 \mathrm{~mm}$. The channel designs were created using a student version of AutoCAD. Figure 6 shows the AutoCAD template design for the two-layer channel study. 


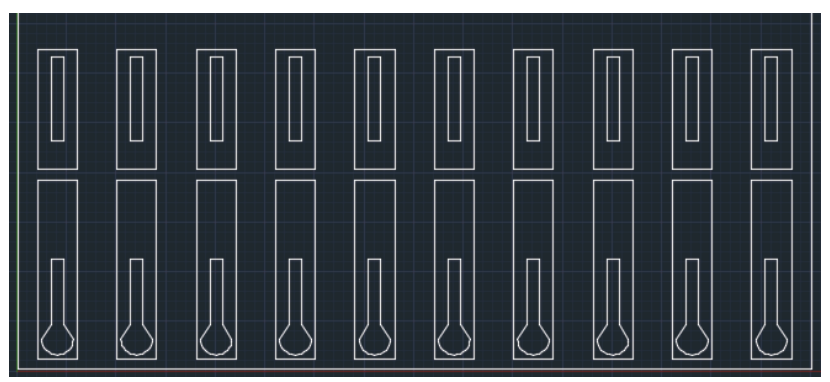

Figure 6: AutoCAD Two-Layer Channel Design

The hydrophobic barriers and hydrophilic channels were created using a wax printing method. The AutoCAD template was plotted and sent to a Xerox Color Cube 8570 wax ink-jet printer, show in Figure 7 below. The designs were printed onto Whatman No. 1 cellulose chromatography paper.

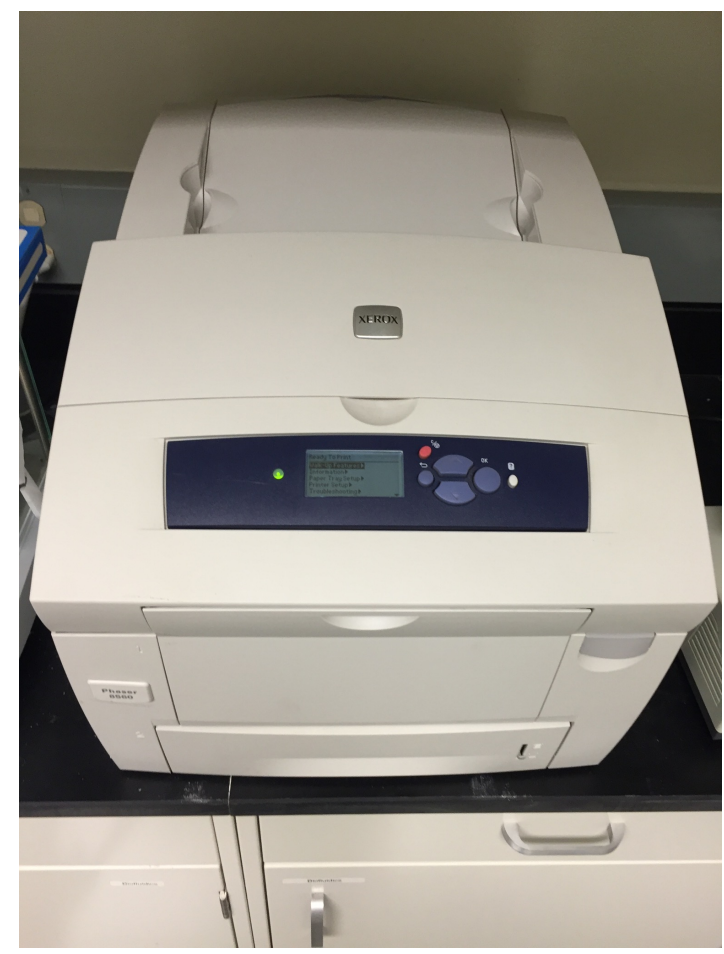

Figure 7: Xerox Color Cube 8570 Wax Printer

The paper was then reheated with a Clarkson digital ceramic top hot plate until the wax fully penetrated the paper. The hot plate was allowed to reach $130^{\circ} \mathrm{C}$ after which 
the paper was placed directly on top for approximately 2 minutes. The hot plate used is shown in Figure 8.

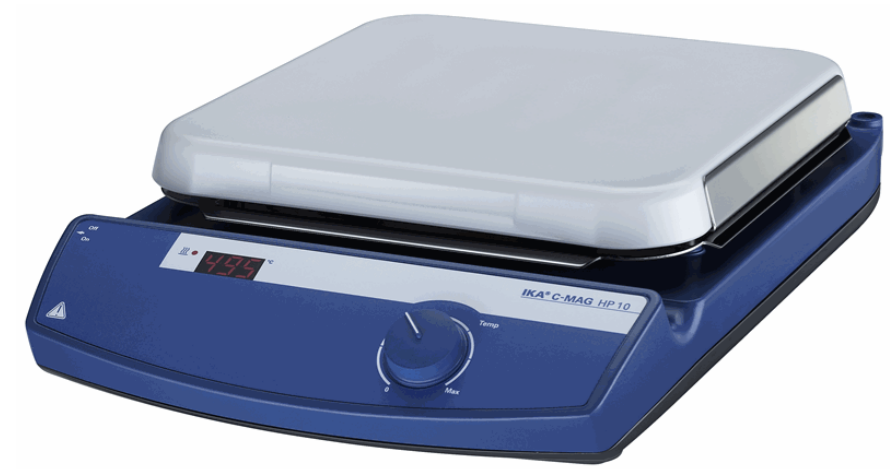

Figure 8: Clarkson Digital Ceramic Top Hot Plate

After two minutes had elapsed the bottom side of the paper was checked for complete melting of the wax. If incomplete melting spots were noticed the paper was placed back on the hot pad until the wax fully penetrated the paper. After complete melting of the wax, the paper was then allowed to cool to room temperature. Each layer was then cut out using a pair of scissors.

\section{Three-layer channel}

The second channel design of the pressure study consisted of three layers. The bottom and the top layers remained unchanged from the previously mentioned two-layer channel design. The new middle layer, also known as the conjugate pad, was designed to have the same channel width with partial overlap of the bottom and top layers. All three layers were designed in AutoCAD and the drawing can be seen in Figure 9. 


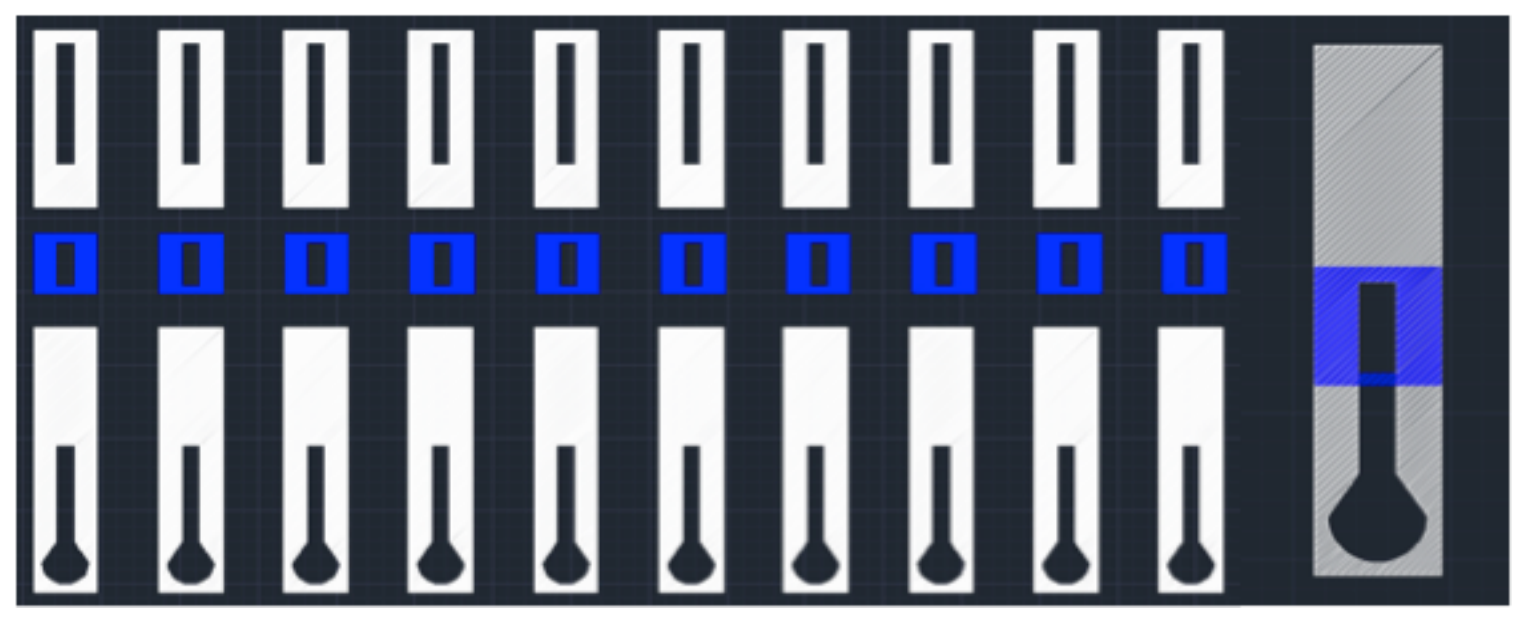

Figure 9: AutoCAD Three-layer Channel Design

Simple pressure housing design

An external housing unit was created for the testing of the two-layer and threelayer channels using AutoCAD. This housing unit was rectangular in shape and consisted of three different pieces that would stack on top of one another. All three pieces had 6 screw holes designed around the perimeter to allow for a bolt to be passed through. The bottom layer was designed as a completely solid layer. The middle layer had a rectangular portion cut out from the center the length and width of the previously designed channels. The top layer was solid expect for a small rectangle that was cut out above the sample input zone.

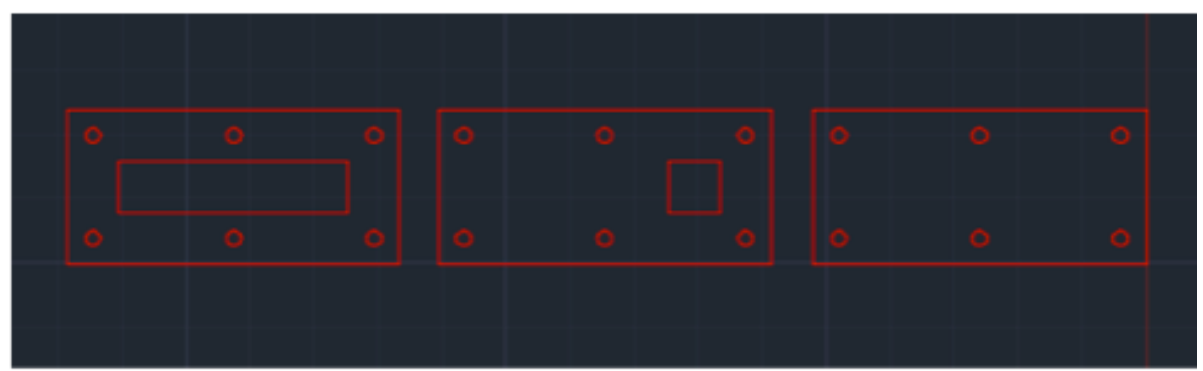

Figure 10: Investigational Pressure Study AutoCAD Housing Design 
The AutoCAD design was then plotted to the laser cutter. Optically clear cast acrylic sheets, $1 / 16$ " thick, were cut using a $\mathrm{CO}_{2}$ Laser cutter. All the cuts were set as single pass vector cuts. After cutting, each piece was cleaned and stored for later use.

\section{Two-layer dye test}

The indirect positive pressure application was first tested on two-layer channel design. The fully assembled device consisted of an isolation layer, two fluid routing layers, the housing unit, and the square rubber O-ring cord stock. To assemble the device, the bottom and middle piece of the housing unit were held together by hand. The isolation layer was first placed into the opening followed by the bottom fluid routing layer then the top fluid routing layer. Two identical length pieces of square O-ring cord stock were then placed adjacent to the overlapping section of the two channels. The top of the housing unit was then put in place and six M4 socket head screws were threaded through the holes and secured in place with nuts.

To visualize the fluid movement, red dye 40 was used as the test sample. Using a $0.2-2 \mathrm{uL}$ range pipette, $1 \mathrm{uL}$ of dye was pipetted into the sample input zone. The dye was allowed to travel through the channel for 5 minutes. If the dye was able to transition from the bottom channel to the top channel the test was marked as pass, otherwise it was marked as fail. Nine replicates of the test were then preformed and the outcome of all ten tests can found in the result section.

\section{Three-layer pressure experiment}

The three-layer experiment was designed to test if the indirect positive pressure would allow for fluid routing through a conjugate pad. Using a 0.2-2 uL range pipette the conjugate pad was loaded with $1 \mathrm{uL}$ of red dye 40 and allowed to dry overnight. The 
device was set up the next day using the same assembly procedure from the two-layer experiment with the exception of the conjugate pad being placed between the bottom and top fluid routing layers.

Using a 0.2-2 uL range pipette $2 \mathrm{uL}$ of deionized water was input into the sample input zone. The dye was allowed to travel through the channel for 5 minutes. If the dye was able to transition from the bottom channel to the top channel the test was marked as pass, otherwise it was marked as fail. Nine replicates of the test were then preformed and the outcome of all ten tests can found in the result section.

\section{3-D Multiplex Platforms (3-D $\mu$ PAD)}

The previous experiments only involved fluid routing through a single channel system. In the next step a 3-D multiplex platform was developed that would allow for simultaneous fluid routing in multiple channels. Based off of the specific aims set forth at the start, fluid would need to be routed through 5 different channels simultaneously. Additionally, fluid would need to be routed to 5 individual conjugate pads, test zones, and control zones. The following details on developing a 3-D multiplex platform are separated into a design section and an experimental section. The design section will be further broken down into the following subsections: multiplex channel design and multiplex housing design.

\section{Multiplex Channel Design 1}

The first channel network design was created from 5 separate layers; an absorbent layer, isolation layer, conjugate layer, fluid routing layer, and a detection layer. The test sample would first enter the channel network in the fluid routing layer. The fluid would then travel along the routing layer and transition down into the conjugate pad layer. The 
fluid would then wick across the conjugate pad, transition back up to the main routing layer and then transition up again to the test zone of the detection layer. After traveling through the test zone the fluid would rout back down to the main routing layer where it would travel a short distance then transition back up to the control zone of the detection. After reaching the end of the control zone, the fluid would transition down through all the layers and into the absorbent pad.

The fluid routing layer was designed with a central sample input zone that connected to 5 different channels. The 5 channels were revolved around the central point creating an equally spaced polar array with an angle of 72 degrees between each channel. The overall length of each channel was approximately $30 \mathrm{~mm}$. The isolation layer was created as an identical copy of the fluid routing layer with the central and subsequent two channels sections removed. Both the fluid routing layer and isolation layer were designed as whole layers.

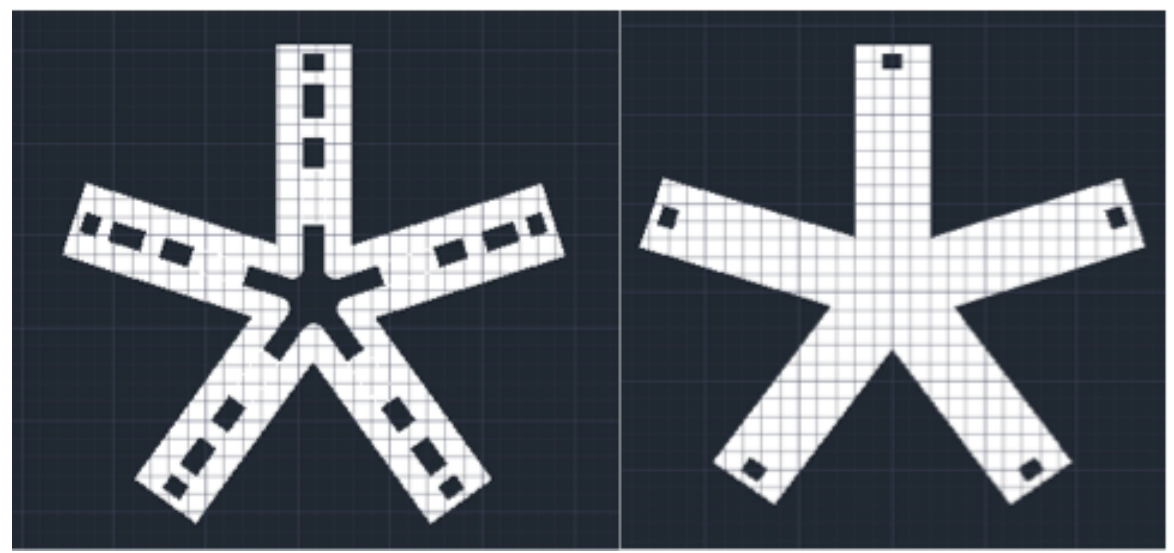

Figure 11: Fluid Routing Layer AutoCAD Design (Left) and Isolation Layer AutoCAD Design (right) 
The conjugate layer and the detection layer were designed as multiple parts rather than a single homogeneous layer, seen in Figure 12 and 13 below. All channels, with the exception of the conjugate pad, had a rectangular geometry.

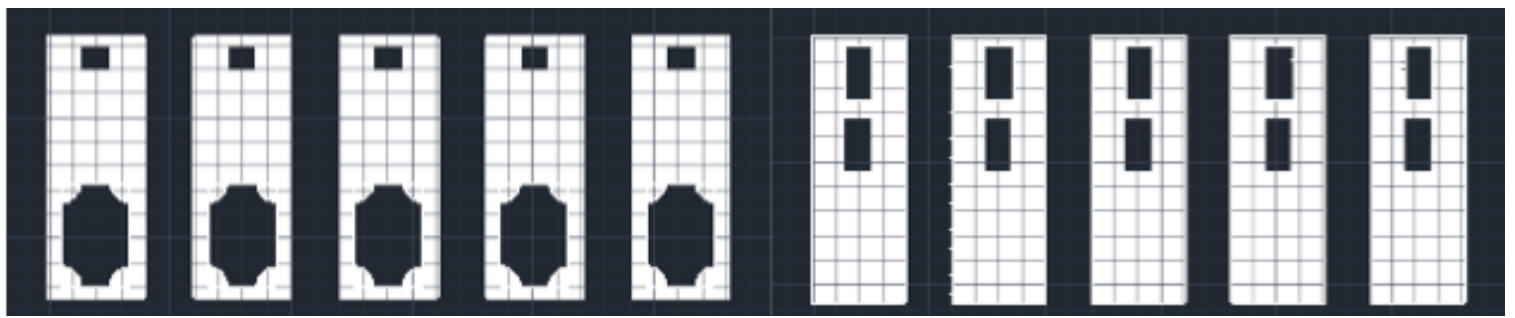

Figure 12: AutoCAD Design of Conjugate Layer (left) and the Conjugate Layer (right)

Additionally, channel widths for all layers were designed to be $2.5 \mathrm{~mm}$ with an overlap of $1 \mathrm{~mm}$ between layers. All patterns were created using a student version of AutoCAD fabricated with the same technique used in the pressure study. Each part was then cut out with scissors and stored for later use. A custom housing unit to fit the channel design was then created.

\section{Multiplex Housing Design 1}

The housing design 1 was created to match the geometry of the channel design 1. The overall shape of the housing unit was a circle. The housing unit consisted of three parts; top, middle, and bottom. The top part had a $8 \mathrm{~mm}$ diameter hole cut in the center. The middle part had a pattern cut out that resembled the geometry of the membrane. The bottom portion was solid. All parts were designed in AutoCAD and designed patterns were cut were cut from $1 / 16$ " thick, optically clear cast acrylic sheets, with a $\mathrm{CO}_{2}$ laser cutter. 
Figure 13: Multiplex Housing Design \#1 AutoCAD Drawing

\section{Multiplex Channel Design 2}

In the second channel design, the isolation layer was the only layer that was

changed. It was made larger by the addition of circular portions connecting to each of the channels.

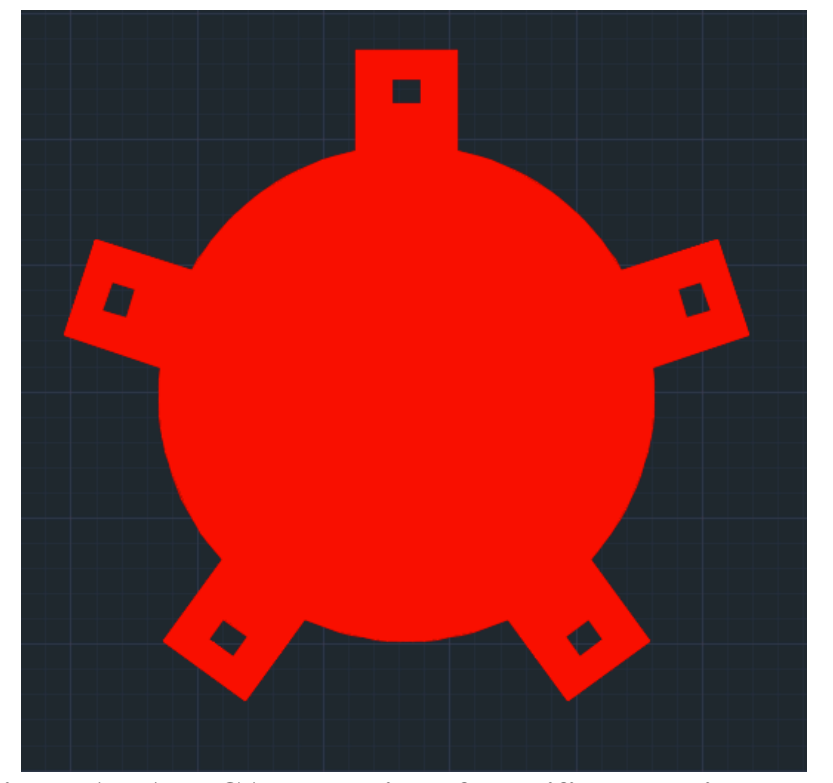

Figure 14: AutoCAD Drawing of Modified Isolation Layer 
The fluid routing layer, conjugate layer, and detection layer remained unchanged from the first design. Channel widths remained $2.5 \mathrm{~mm}$ and the overall length of the channel remained $30 \mathrm{~mm}$. Again as in the previous design, the isolation and fluid routing layer were designed as whole layers. Although shown here in the assembled format, the conjugate and detection layers were designed as individual parts.

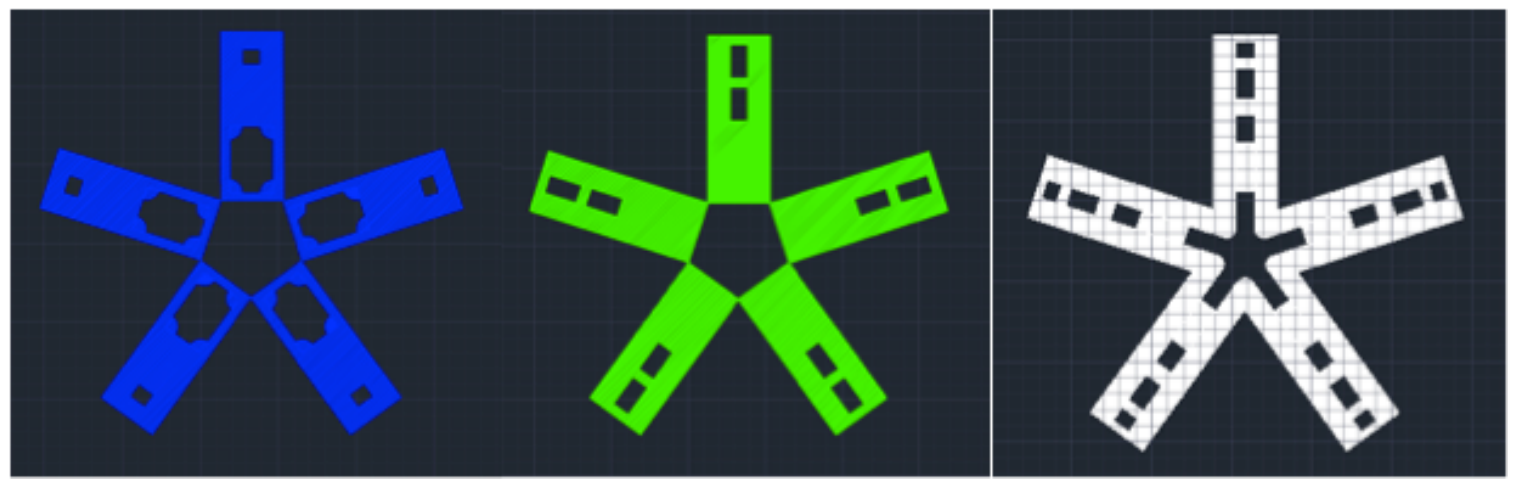

Figure 15: AutoCAD Drawing of Conjugate Layer (left), Detection Layer (middle), Fluid Routing Layer (right)

\section{Multiplex Housing Design 2}

The modification of the isolation layer required a new housing design. The middle section of the housing unit was changed and modeled after the geometry of the new isolation layer. Since the middle section was the only section that changed, the top and bottom sections from the first housing design were kept the same. 
Figure 16: Multiplex Housing \#2 AutoCAD Drawing. Bottom section (left), middle section (center), top section (right)

\section{Multiplex Channel Design 3}

Multiple changes were made to the channel layout in the third channel design. All of the layers were designed within equal size circular regions. There were more individual parts of layers; each layer was designed as a whole. For the fluid routing layer, the width of the channels remained the same however the ends of each channel were rounded and the overlap between layers was increased to $2 \mathrm{~mm}$. The geometry of the conjugate pad region remained the same and all 5 pads were designed on a single layer. The edges of the test and control regions of the detection region were rounded but elsewise remained the same. Instead of a small rectangle at the end of the channels, a ring of open region was designed to allow for fluid to rout from the end of the control region to the absorbent pad. 


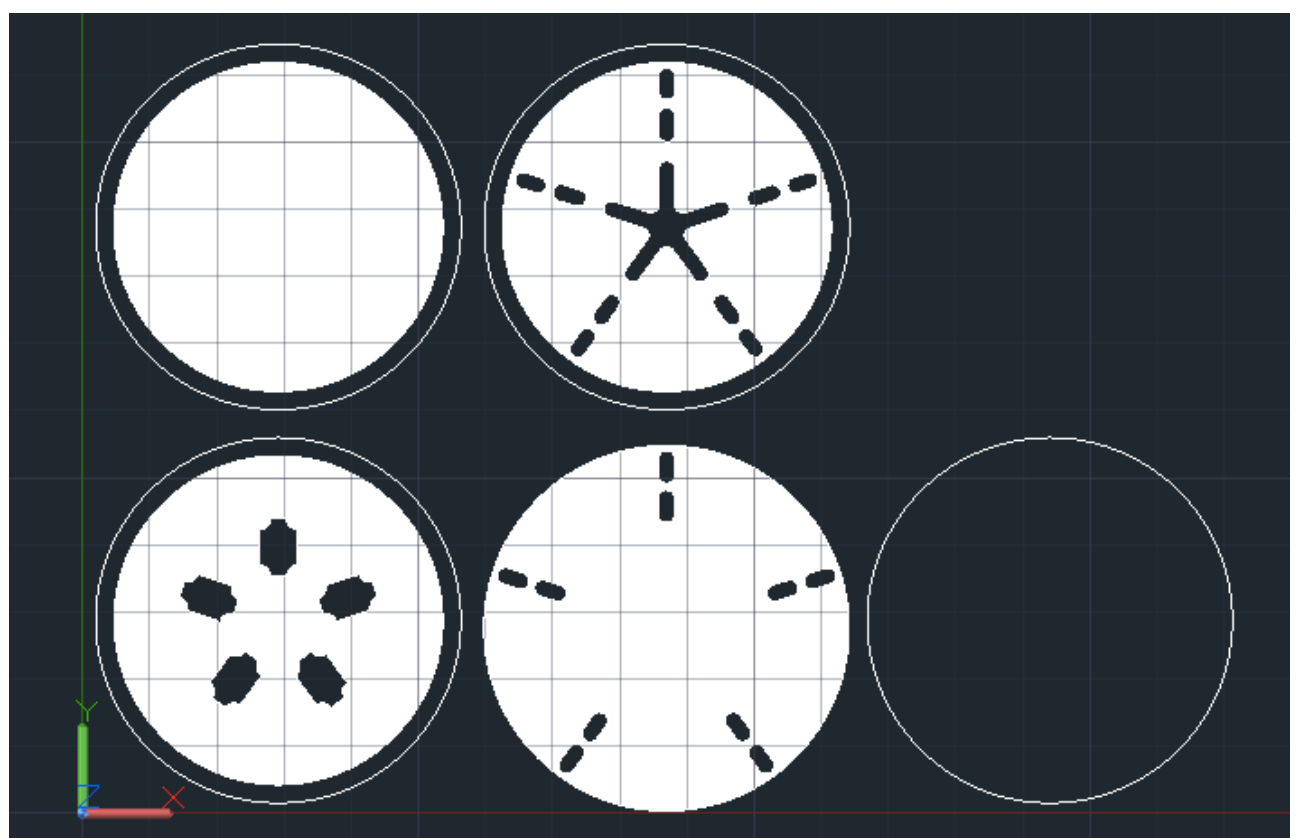

Figure 17: AutoCAD Drawing of Channel Network Design \#3 Top: Barrier Layer, Fluid Routing Layer. Bottom: Conjugate Layer, Detection Layer, Absorbent Layer

\section{Multiplex Housing Design 3}

The third housing design also was created from three pieces. Only the bottom section was the same as in the previous designs the middle and top pieces were modified. The middle piece of the housing unit had a circular portion the same diameter as the layers cut out from the center. The top layer had the same hole cut out from the center for the sample input but also had the addition of groves. The groves were cut on the interior side of the top section to allow for the placement of the rubber cord stock. The groves were etched using the $\mathrm{CO}_{2}$ laser cutter with the settings modified to produce a partial cut into the acrylic sheet. All housing components were cut from 1/16" optically clear cast acrylic sheets. 
Figure 18: Multiplex Housing Design \#3 AutoCAD Drawing

\section{Multiplex Channel Design 4}

Similar to the previous design, equal sized circular layers were used as templates.

The shape of the conjugate pad was changed to an oval shape. The AutoCAD channel design width was increased to $3.65 \mathrm{~mm}$ for all layers. The overlap between layers was increased to $3 \mathrm{~mm}$. Additionally; three alignment holes were added to the fluid routing layer, conjugate layer, and detection layer.

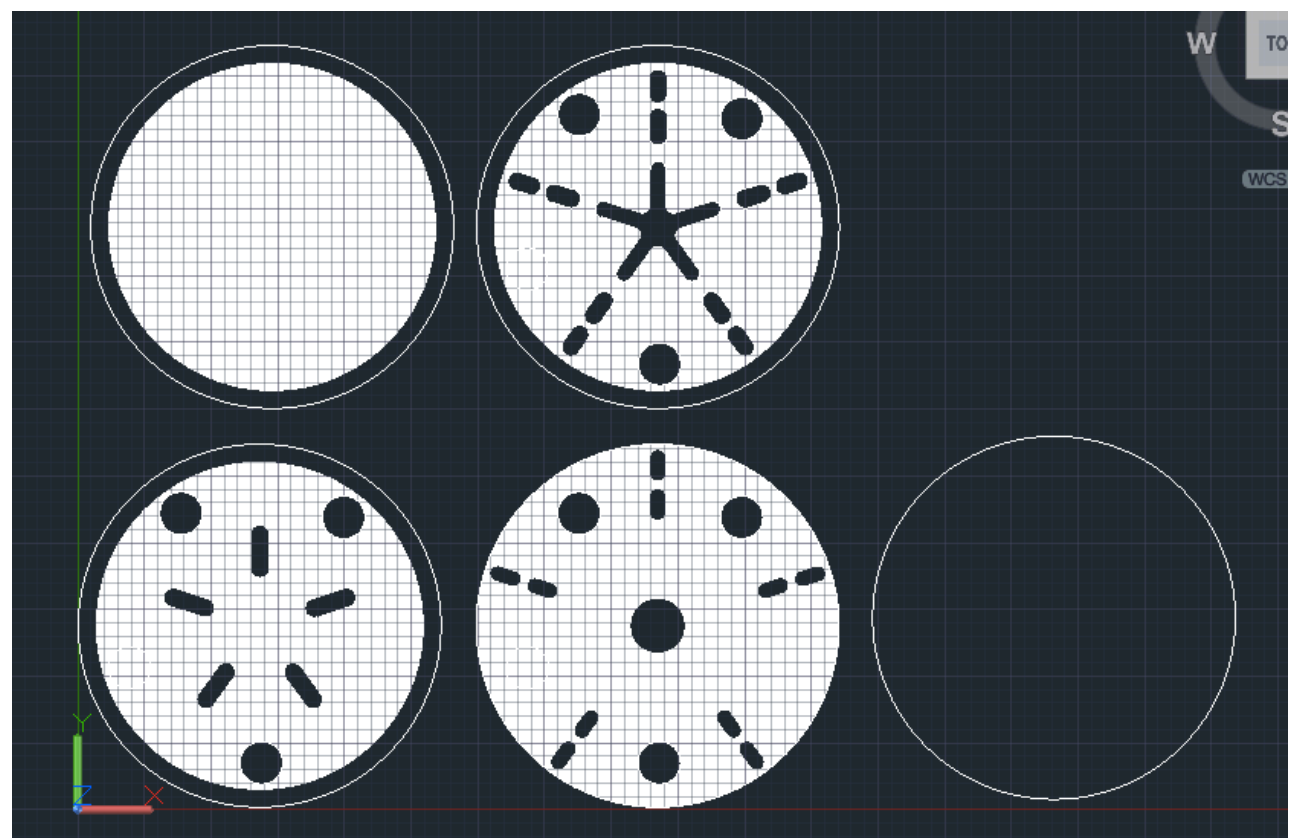

Figure 19: Multiplex Channel Network Design \#4. Top: Barrier Layer, Fluid Routing Layer. Bottom: Conjugate Layer, Detection Layer, Absorbent Layer 


\section{Multiplex Experimental Section}

The following experimental subsections include the demonstration of a sample solution routing through multiple layers of membrane using indirect positive pressure, as well as the resolubilization and routing of a glucose detector reagent.

\section{Multiplex Dye Experiment}

All channel designs with their respective housing units were tested for the ability to rout fluid from the input of the device to the end of each channel. The layers of each device were fabricated prior to testing with the wax printing method previously discussed. Using a $2-20 \mu \mathrm{L}$, range pipette $1 \mu \mathrm{L}$ of red dye 40 was deposited in the reagent zones of the conjugate layer. The dye was then dried at room temperature for 1 hour. The device was then assembled according to its respective protocol. A 20-100 $\mu \mathrm{L}$ range pipette was used to introduce $50 \mu \mathrm{L}$ of deionized water to the sample input. The solution was allowed to wick through the $\mu \mathrm{PAD}$ for 5 minutes. The development time, defined as the time at which the leading edge of the fluid contacted the end of the control zone, was recorded for each of the five channels. Dye testing was repeated for all channel designs in the same manner. Four additional replicates were performed for channel designs \#1-3 and an additional nine replicates were performed for channel design \#4. The channel development time results were evaluated on JMP using an ANOVA test.

\section{Multiplex Glucose Experiment}

Channel design \#4 and housing design \#3 were selected as the designs to be testing with a colometric glucose assay. A reagent pencil containing $2 \% \mathrm{w} / \mathrm{w}$ glucose oxidase (200 U/mg), $2 \% \mathrm{w} / \mathrm{w}$ horseradish peroxidase $(67 \mathrm{U} / \mathrm{mg})$, and 10\% w/w ABTS was used to deposit reagents onto the top side of the reagent zones of the conjugate layer. 
The housing unit was assembled and secured in place by hand tightening of the bolts. Test solutions (50 $\mu \mathrm{L})$ containing glucose in concentrations ranging from $0 \mathrm{mM}$ to 1.2 $\mathrm{mM}$ prepared in $1 \mathrm{XPBS}$ were added to the sample zone. The solutions were allowed to wick through the layers and were dried for 30 minutes before layers were removed from the housing unit and inspected. 


\section{RESULTS}

\section{Investigational Pressure Testing}

The results from the investigational pressure study, the dye testing, and the glucose concentration testing are presented in this section. Table 4 and 5 present the results from the two-layer and three-layer investigational pressure study.

Table 4: Two-Layer Pressure Test

\begin{tabular}{|c|c|c|c|c|c|c|c|c|c|c|}
\hline Test & 1 & 2 & 3 & 4 & 5 & 6 & 7 & 8 & 9 & 10 \\
\hline Result & Pass & Pass & Pass & Pass & Pass & Pass & Pass & Pass & Pass & Pass \\
\hline
\end{tabular}

Table 5: Three-Layer Pressure Test

\begin{tabular}{|c|c|c|c|c|c|c|c|c|c|c|}
\hline Test & 1 & 2 & 3 & 4 & 5 & 6 & 7 & 8 & 9 & 10 \\
\hline Result & Pass & Pass & Pass & Pass & Pass & Pass & Pass & Pass & Pass & Pass \\
\hline
\end{tabular}

\section{Dye Testing}

The results from the dye testing are presented in the following tables below. All development times are presented in seconds. Channels that have an N/A indicate that fluid was unable to reach the control zone. Channels that have an INC indicate that dye was present in the control zone but was not able to reach the end of the zone.

The results for testing of device \# 1 are shown in Table 6 below. This device was made from the combination of channel design \#1 and housing unit \#1.

Table 6: Device \#1 Dye Test Development Times

\begin{tabular}{|c|c|c|c|c|c|}
\hline & \multicolumn{5}{|c|}{ Development Time (s) } \\
\hline Channel \# & Test 1 & Test 2 & Test 3 & Test 4 & Test 5 \\
\hline 1 & N/A & N/A & 86 & 110 & 109 \\
\hline 2 & N/A & N/A & N/A & N/A & 84 \\
\hline 3 & N/A & 100 & N/A & 264 & N/A \\
\hline 4 & N/A & N/A & 94 & N/A & N/A \\
\hline 5 & N/A & N/A & 127 & N/A & N/A \\
\hline
\end{tabular}


The results for testing of device \# 2 are shown in Table 7 below. This device was made from the combination of channel design \#2 and housing unit \#2.

Table 7: Device \#2 Dye Test Development Times

\begin{tabular}{|c|c|c|c|c|c|}
\hline & \multicolumn{5}{|c|}{ Development Time (s) } \\
\hline Channel \# & Test 1 & Test 2 & Test 3 & Test 4 & Test 5 \\
\hline 1 & 143 & 141 & N/A & 170 & N/A \\
\hline 2 & N/A & 103 & 204 & N/A & N/A \\
\hline 3 & N/A & N/A & 220 & 130 & N/A \\
\hline 4 & 93 & 109 & N/A & N/A & N/A \\
\hline 5 & N/A & 140 & 84 & N/A & N/A \\
\hline
\end{tabular}

The results for testing of device \# 3 are shown in Table 8 below. This device was made from the combination of channel design \#3 and housing unit \#3.

Table 8: Device \#3 Dye Test Development Times

\begin{tabular}{|c|c|c|c|c|c|}
\hline & \multicolumn{5}{|c|}{ Development Time (s) } \\
\hline Channel \# & Test 1 & Test 2 & Test 3 & Test 4 & Test 5 \\
\hline 1 & 166 & INC & 127 & 173 & 114 \\
\hline 2 & INC & 142 & 120 & INC & 211 \\
\hline 3 & INC & INC & 127 & 143 & 156 \\
\hline 4 & 237 & 191 & 127 & N/A & 122 \\
\hline 5 & 92 & 153 & 120 & 109 & 92 \\
\hline
\end{tabular}

The results for testing of device \# 4 are shown in Table 8 below. This device was made from the combination of channel design \#4 and housing unit \#3. 
Table 9: Device \#4 Dye Test Development Times

\begin{tabular}{|c|c|c|c|c|c|c|c|c|c|c|}
\hline & \multicolumn{10}{|c|}{ Development Time (s) } \\
\hline $\begin{array}{c}\text { Channel } \\
\#\end{array}$ & Test & Test & Test & Test & Test & Test & Test & Test & Test & Test \\
\hline 1 & 180 & 146 & 197 & 4 & 5 & 6 & 7 & 8 & 9 & 10 \\
\hline 2 & 103 & 92 & 79 & 107 & 162 & 151 & 135 & 141 & 140 & 110 \\
\hline 3 & 149 & 117 & 118 & 131 & 118 & 171 & 113 & 174 & 153 & 118 \\
\hline 4 & 149 & 122 & 128 & 127 & 123 & 144 & 150 & 196 & 154 & 135 \\
\hline 5 & 167 & 105 & 112 & 101 & 89 & 128 & 92 & 123 & 91 & 92 \\
\hline
\end{tabular}

The mean development time for each channel is shown in Figure 20 below. Using an alpha value of 0.05 , the results from the ANOVA test indicated a significant difference between channel 5 and channels 1 and 3 .

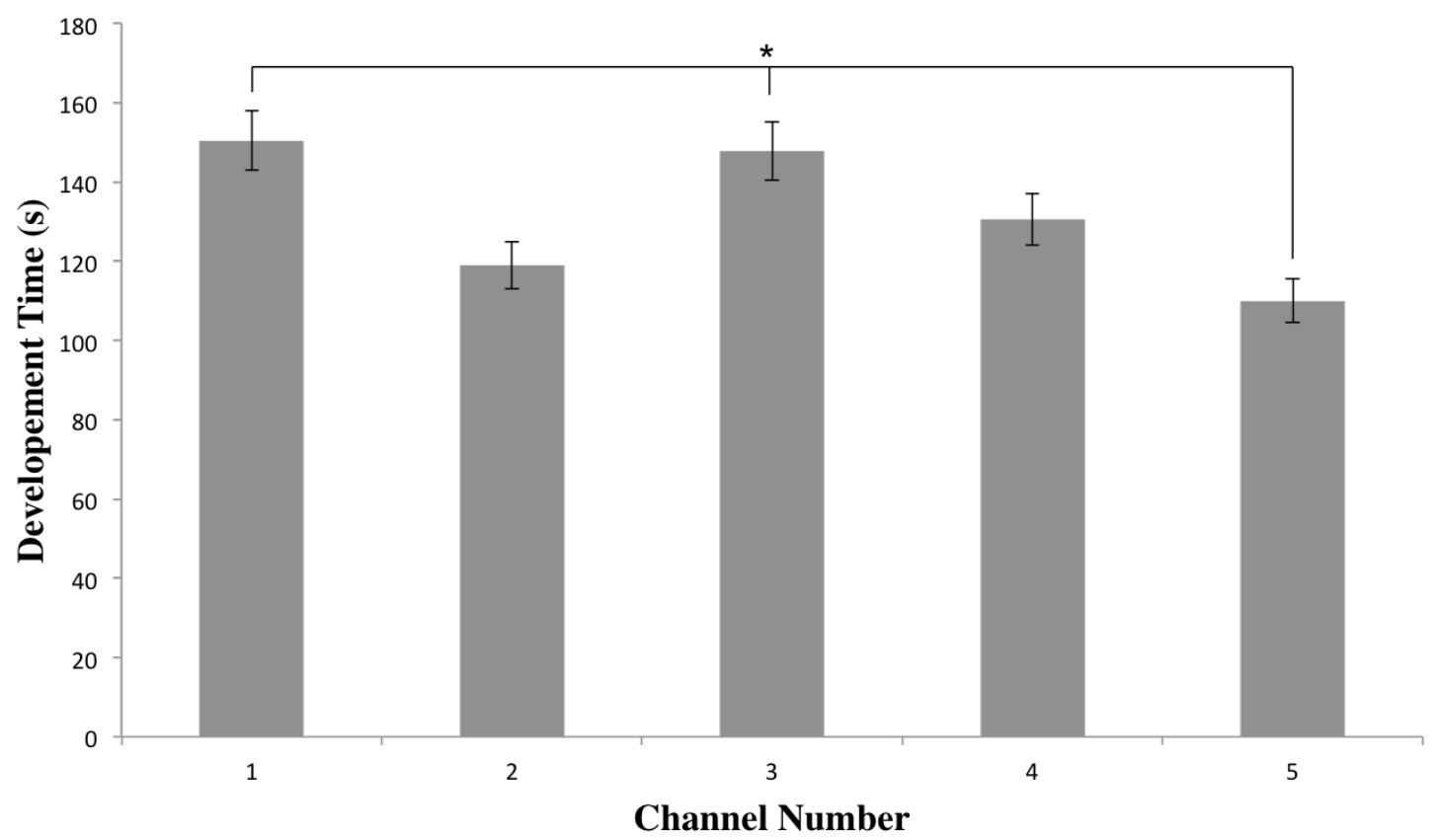

Figure 20: Mean channel development time from dye testing of device \#4. Development times are reported in seconds. (* Indicates a significant difference in the mean channel development time between channel 5 and channels 1 and $3(p<0.05)$.) 


\section{Glucose Testing}

The results from the glucose concentration testing are listed in Table 10 below. The result, either a Yes or No, was recorded for the presence of a blue/green signal in either the test or control zone.

Table 10: Glucose Concentration Test Results

\begin{tabular}{|l|l|l|l|l|}
\hline Glucose Concentration & $1.2 \mathrm{mM}$ & $0.6 \mathrm{mM}$ & $0.3 \mathrm{mM}$ & $0 \mathrm{mM}$ \\
\hline Test 1 & Yes & No & No & No \\
\hline Test 2 & Yes & No & No & No \\
\hline Test 3 & Yes & No & No & No \\
\hline Test 4 & Yes & & & \\
\hline Test 5 & Yes & & & \\
\hline
\end{tabular}

Additional pictures of the glucose concentration test can be found in the discussion section of this report. 


\section{DISCUSSION}

The following section contains the discussion of the investigational pressure study, the dye testing for each of the device iterations, and the glucose concentration testing.

\section{Investigational Pressure Study}

The investigational pressure study was performed to determine if applying pressure along the edge of the channel with the rubber cord stock would allow for fluid to transition vertically across layers. Simple channel designs along with a housing unit were created for this test. The two-layer design was tested first by pipetting a dye solution into a channel on the bottom layer and observing if the fluid was able to rout into the top layer. All ten tests done with the two-layers were successful in routing fluid from the bottom layer to the top layer.

The same channel design and housing unit, along with the addition of a third layer were tested next. In this test the dye was deposited onto the middle layer and allowed to dry. This was to simulate a conjugate pad that would be storing reagents. The three-layer design was again tested in the same manner. It was found that the fluid was able to wick from the bottom layer into the middle layer where it would solubilize the dye before routing up to the top layer. This test confirmed that the rubber cord stock could be used to apply pressure along the edge of the channel to allow for fluid conductivity between layers. More complicated designs could now be made using this pressure application. 


\section{Device \#1}

Table 6 shows the results of the dye test for device \#1. All tests were seen to have at least one or more channels where fluid was unable to rout into both test and control zones. The first test had the worst performance in that it was only able to route fluid into a single test zone (Fig. 21). The subsequent tests showed improvement in fluid routing, however complete development of all channels was not achieved. The second and fourth tests had successful fluid routing in all five test zones but limited success in the control zones. Fluid was observed in only one of the control zones in the second test and two in the fourth test. The third and fifth tests had successful fluid routing in some of the channels but were unable to achieve routing in all five channels. Only three channels fully developed in the third test and two in the fifth test. Upon completion of each test, the device was disassembled and the layers were inspected to determine the cause of failure for the undeveloped channels.

The individual layers showed that fluid was able to wick from the sample input and down into the conjugate pad. The cause of failure in most cases was the transition between the end of the reagent zone and the fluid routing layer below. In the tests where the fluid was unable to reach the test zone, it was noticed that there was little to no overlap between the end of the conjugate pad layer the fluid routing layer. Figure 21 shows the topside of the fluid routing layer from the first test. The residual dye solution from the test shows that the conjugate layer stopped short of overlapping the fluid routing layer in four of the five channels. With no overlap present, it would be impossible for fluid to continue through the channel. In other tests where dye was only seen in the detection zone, again it was noticed that there was a lack of an overlap between layers. 


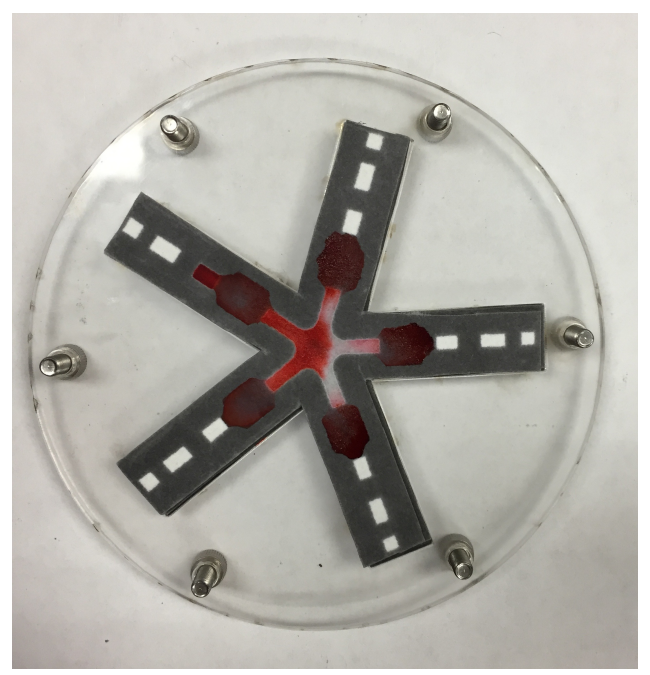

Figure 21: Top view of the fluid routing layer from the first dye test of device \#1 showing the lack of overlap between the conjugate layer and the fluid routing layer.

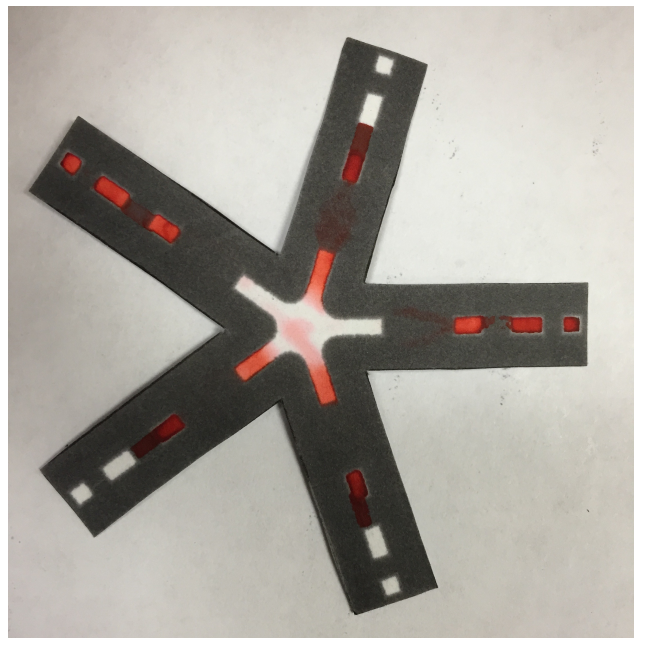

Figure 22: Top view of the fluid routing layer from dye test showing the misalignment of a layer in channel 2.

The two main sources for error were identified as either the initial design or the assembly process. Since some of the channels had fluid successfully routed through both zones it would seem that the cause of the lack of overlap in some channels could be attributed more so to the assembly process. The variation in channel development is most 
likely due to the patterns being cut out after the wax was melted as the melting process causes the edges of the wax to spread outward resulting in a loss of precision when cutting along the edge.

It was noticed that the cut made on the outer edge of the conjugate and detection layers had the most influence on channel development. In most cases the outer edge was undercut causing the channels to shift inward. Since only the conjugate or detection layer was shifting while the fluid routing layer remained unchanged this would cause the overlap decrease or in some cases become nonexistent. The initial design of the overlap between layers accounted for the spreading of the wax during the melting process however it was assumed that the outer edge would remain fixed. Since the outer edge was used to line up the channels it was critical that the edge was cut in the proper place.

It was also noticed that the assembly process of this design was very tedious. The absorbent pad, isolation layer, and fluid routing layer was slightly challenging to cut out. Additionally, since the conjugate pad and the detection layers were designed as individual parts of each channel it required a cumbersome number of parts to cut out by hand. There were 13 parts in total that were required to be cut out to assemble a complete device. During the assembly process, it was found to be difficult to get all the layers, especially the individual pieces of the conjugate and the detection layers to stay in place before the top of the housing component could be put into place. This increased both the time it took to assemble the device as well as the chance for misalignment of the layers. Although complete development of all channels was not accomplished, these experiments did show the indirect pressure application with the rubber cord stock was effective in promoting fluid connectivity between layers. If the channels were in the 
correct positions with some overlap between layers the device would function

successfully. These initial experiments show promise for the use of rubber cord stock to apply indirect pressure in a multi-layer micro-PAD.

\section{Device \#2}

Table 7 shows the results of the development times for channel and housing design \#2. Similar problems that had occurred during testing of device \#1 were present for device \#2. In all tests, there was unsuccessful channel development in at least one or more channels. The second test had the most successful results with four fully developed channels. The third test saw complete development in three channels. The first and fourth had development in two channels. The fifth test was unable to rout fluid to any of the channels. It was noticed that different channels in different tests were able to rout fluid through the entire channel. This was the same issue that was present with the first device.

Post-test inspection revealed that the main issue was with the conjugate pad and the fluid routing layer. As was the case with the first device, there was a discrepancy with the degree of overlap or lack there of between layers. Again this was attributed to the patterns being cut out after the wax had been melted. The design overlap distance may have been too short given the amount of variation present in the current hand assembly process.

Additionally, the assembly process for device \#2 was found to be more cumbersome than for the first device. The lack of guiding walls along the sides of each channels made the smaller individual layers (conjugate and detection) more prone to shifting side to side. It was difficult to get all layers to line up and remain in the proper positions. The assembly process may be able to be improved with the addition of glue or 
tape to hold the layers together. However, it was thought that there could be a way to accomplish accurate alignment without the need for any adhesives.

\section{Device \#3}

Development times of all channels from each test with device \#3 are shown in Table 8. Each test had successful fluid routing in all five test zones however, there was variable success in the control zones. A total of two tests had successful development of all channels while the remaining three had issues at least one channel.

In both the first and second tests, there was successful development of three channels and incomplete fluid routing in the remaining two channels. Incomplete fluid routing was where the dye could be seen in beginning part of the control zone but was not able to reach the end of the zone. Incomplete fluid routing was found in channels 2 and 3 in the first test and channels 1 and 3 in the second test. In the first test, successful channel development times were 92 seconds (channel 5), 166 seconds (channel 1), and 237 seconds (channel 4). In the second test, successful development times were 142 seconds (channel 2), 153 seconds (channel 5), and 191 seconds (channel 4).

The fourth test had successful fluid routing in channels \#1, 3, and 5, incomplete fluid routing in channel \#2, and unsuccessful fluid routing in the control zone of the channel \#4. For the channels that were successful, the development times were 109 seconds (channel 5), 143 seconds (channel 3), and 173 seconds (channel 1). Out of all the tests with device \#3, this was the only instance where fluid was unable to rout into the control zone of a channel. The result of the fourth test is shown in Figure 23 below. 


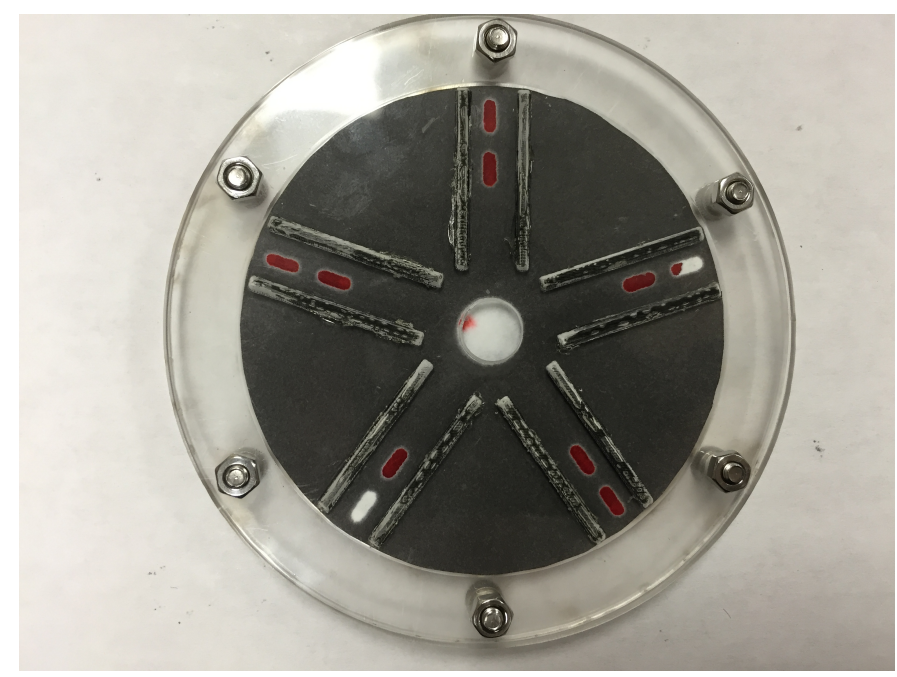

Figure 23: Results from the fourth dye test. Channel 2 was observed to have incomplete fluid routing and channel 4 had unsuccessful fluid routing.

The third and fifth tests were able to successfully rout fluid through all five channels shown in Figure 24 below. The development times for the third test were nearly identical for each channel. There was a maximum difference of 7 seconds between the first and last channels that fully developed at 120 seconds and 127 seconds, respectively. The development times of the fifth test were more varied. The first channel fully developed after 92 seconds while the last channel fully developed at 211 seconds. The remaining development times were 114 seconds, 122 seconds, and 156 seconds. 

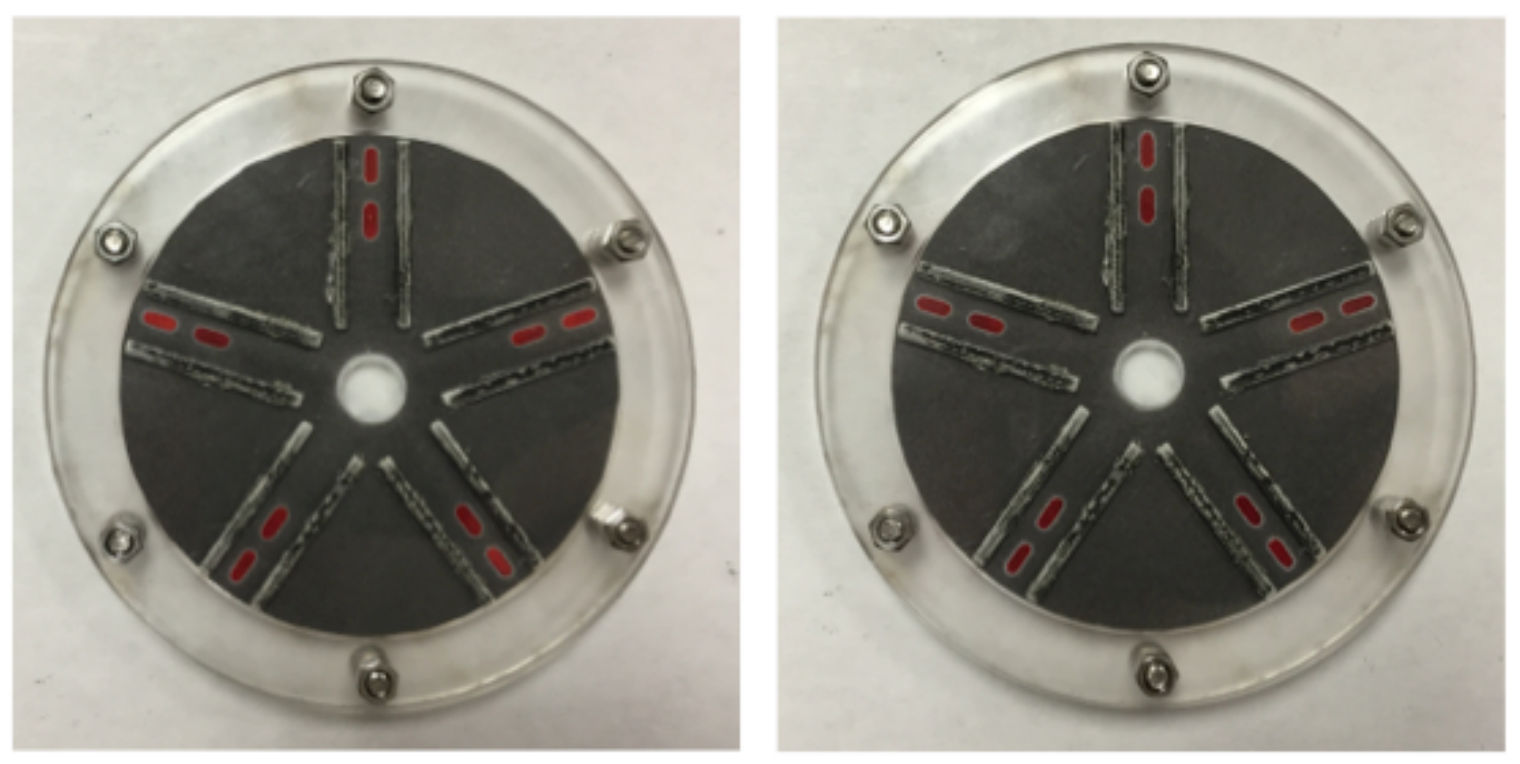

Figure 24: Dye test results from the third test (left) and fifth test (right).

According to the test protocol, the determination of incomplete was made after five minutes had elapsed since the start of the test. The devices continued to be observed for additional time with no change noticed in the incomplete channels. This suggests that the solution had reached equilibrium within the device and would no longer continue to wick via capillary force. While the exact time at which fluid flow ceases is unknown, under the particular circumstances of this test the device run time can be assumed to be less than 5 minutes. Since the results were recorded after the fluid flow had ceased, the incomplete channel determinations were therefore due to a shortage of fluid volume in those channels rather than a lack of time.

Inspection of the used layers showed that in some cases the alignment of the channels between layers was slightly off. This caused a slightly uneven flow pattern of the fluid when it initially enters the detection and control region. Ideally, the leading edge of the fluid would travel perpendicular to the channel walls throughout the entire length 
of the channel. However, the uneven flow was able to self-correct as the fluid traveled throughout the zone.

It was noticed in all but the third test, that when the sample was pipetted into the input region a portion of the liquid would form a dome on top of the layer. This would cause the input region of the top layer to balloon up and lose contact with the layer below. It's possible that the lack of physical connection between the two layers is responsible for the difference in development success as well as times in each channel.

Additionally, it was noticed that the modification of the layers to a circular geometry resulted in a reduced assembly time. The number of pieces that required cutting dropped from 13 to 5 . The reduction in individual pieces also made assembly of the device an easier process. Ultimately, the results from testing show that the channel and housing design have improved from the previous designs. However, improvements in the housing design and layer alignment will need to be made to improvement the overall functionality of the device.

\section{Device \#4}

The results of the dye test for device \#4 are shown in Table 9. On average, channel 5 had a development time of (110s), followed by channel 2 (119s), channel 4 (130.6), channel 3 (147.8s), and channel 1 (150.4s). The mean channel development times can be seen in Figure 20 in the Results section. Evaluation of the channel development times using an ANOVA test in JMP indicate that there is evidence that the mean development time for each channel is not equal $(\mathrm{F}(4,45), \mathrm{p}=0.0024)$. The residuals were checked with a Goodness of Fit test and appeared normally distributed $(p=0.33)$. A Bartlett test was then conducted confirming the homogeneity of variance of the residuals 
$(\mathrm{p}=0.324)$. A post-hoc power analysis indicated a power of greater than $90 \%$. A comparison of the means using a Tukey-Kramer HSD test suggest that only channels 1 and 3 are significantly different from channel 5 . This was expected as channel 5 had the fast development time, while channels 1 and 3 had the slowest development times. More testing will need to be conducted to determine if the difference in development times is a product of the housing or channel design. A result from the sixth dye test can be seen in Figure 25 below.

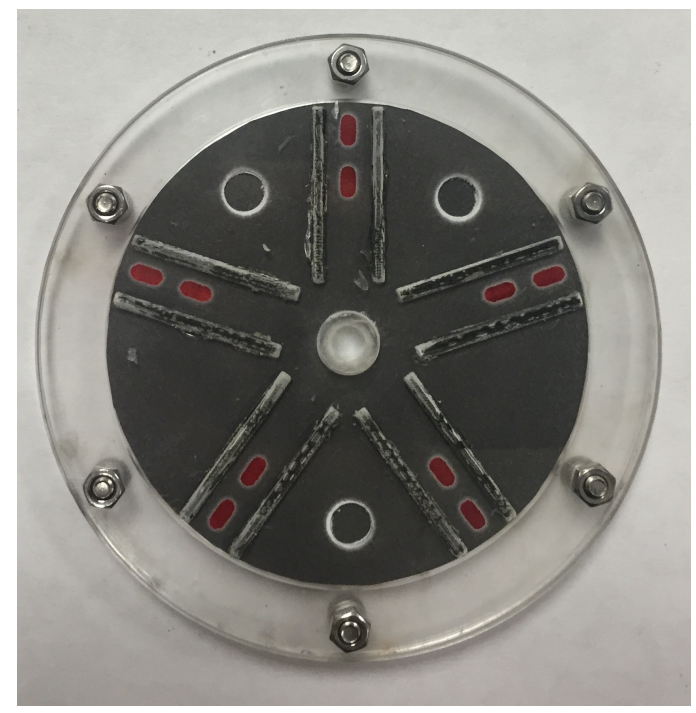

Figure 25: Result from the sixth dye test. Also shown are the three alignment holes and the rubber tubing.

It wasn't noticed until the third device that the channel widths were not as wide as intended. The previous designs had estimated that the width would decrease by $0.55 \mathrm{~mm}$. On average, the channel widths were measured at $1.9 \mathrm{~mm}$ indicating a decrease in width of $1.15 \mathrm{~mm}$. The channel design widths in AutoCAD were therefore increased from $3.05 \mathrm{~mm}$ to $3.65 \mathrm{~mm}$. The increased design width resulted in actual channels widths closer 
to the expected $2.5 \mathrm{~mm}$. Due to the small sample size from pervious tests, it's unclear if smaller channel widths had an affect on the development times.

The addition of the alignment holes to the layers made an improvement during the assembly process. The irregular fluid flow that was noticed with the previous device was absent in device \#4. Inspection of the used layers also confirmed that the channels were aligned properly. During the assembly process, it was noticed that there was some slight rotating of the layers as they were placed into the housing unit. The misalignments were corrected before the test was run however; this could be eliminated by the addition of pegs that the alignment holes of each layer would be placed over.

The rubber tubing added to the sample input hole of the housing unit was thought to have the most impact of the design changes in terms of performance. The tubing served a twofold purpose, in that: 1) it served as a reservoir for the sample input and 2) it prevented the top layer from ballooning up when the sample was applied and allowed for a more consistent connection between the detection layer and the fluid routing layer. It's thought that in future designs the tubing could be replaced with a larger reservoir that would allow for samples sizes of greater volume.

Device \#4 had the most promising results of any of the previously tested designs. There was successful development of all channels in every test. This was a major improvement over the previous designs put forth in this thesis.

\section{Glucose Test}

The results from the dye testing suggested that the fourth channel design was capable of consistently routing fluid through all layers and was therefore the best 
candidate for further testing. A colorimetric glucose assay was used as the model test to demonstrate the ability of the device to detect a specific analyte in a solution. This assay relies on a coupled enzymatic reaction, which ultimately results in the oxidation of ABTS from a colorless reduced form to a blue-green oxidized form. An important distinction to note about this assay is that the reagents were deposited via a reagent pencil created by Martinez et. al. This custom made pencil contains reagents stored in a solid matrix and allows for quick, easy, and solvent-free deposition of reagents onto a paper membrane. To date, this is the first known use of a reagent pencil used in conjunction with a multilayer micro-PAD.

Table 10 shows the results of the glucose testing. All tests using the $1.2 \mathrm{mM}$ glucose concentration resulted in a positive identification. The tests with $0.6 \mathrm{mM}, 0.3 \mathrm{mM}$ concentrations of glucose were not able to produce a visual signal. Additionally, all tests with a $0 \mathrm{mM}$ concentration had no visual signal. Figure 26 shows an example of the visual results from the third $1.2 \mathrm{mM}$ concentration test. 


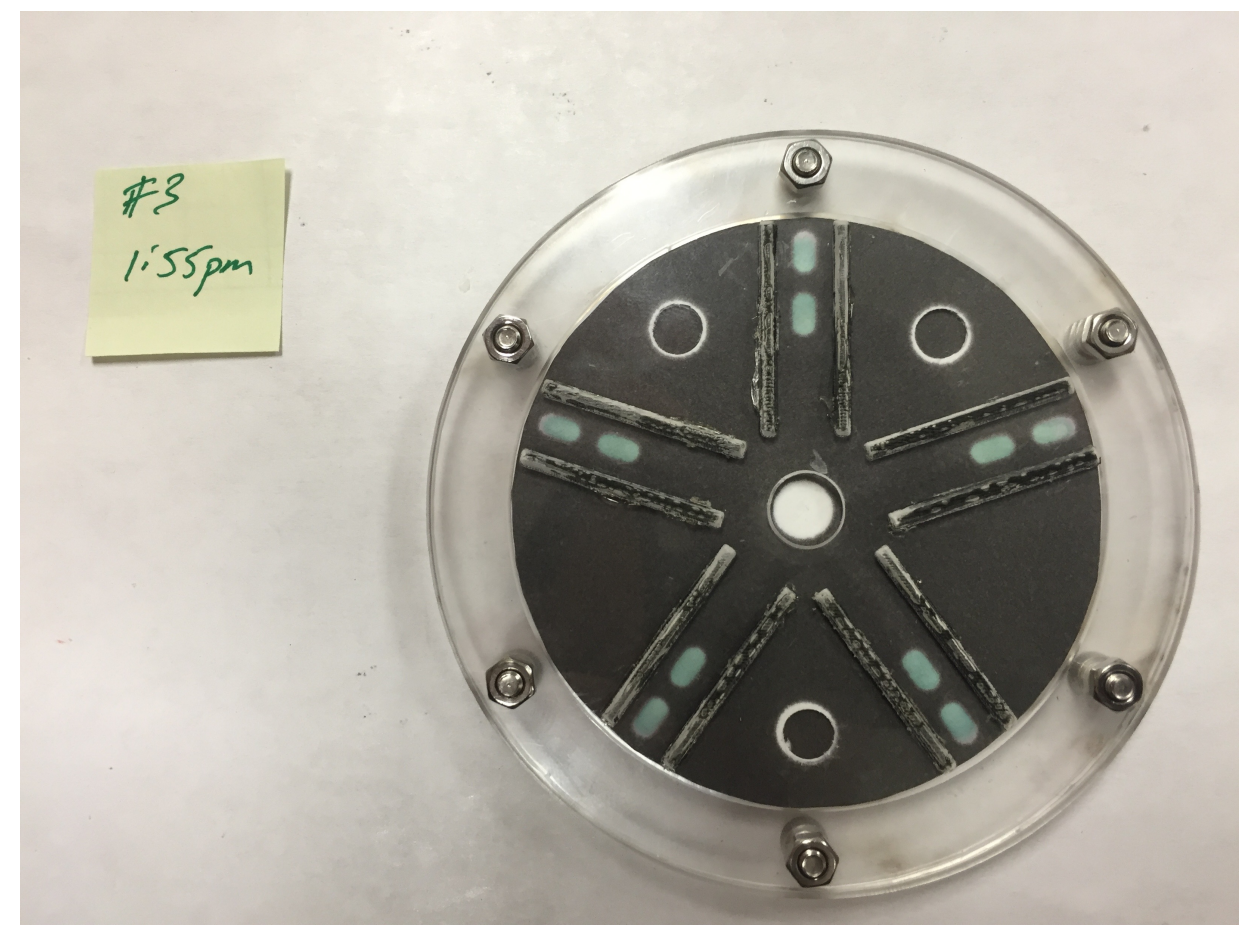

Figure 26: Result from the 1.2mM glucose concentration testing. Blue/green color indicates the presence of glucose.

The channel design remained the same with the exception of the fluid routing layer where the outer transition ring was filled with wax. This was to prevent the fluid from transitioning out of the control zone. It was necessary to halt fluid flow at the end of the control zone because there was no mechanism for immobilizing the reagents after they were released from the conjugate layer. If left unchecked, the oxidized ABTS could potentially wick down to the lower layers which resulting in a weaker visual signal

In all of the glucose concentration tests, the reagents were deposited only on the top of the conjugate layer. Figure 27 shows the reagents that were deposited before some of the tests. The top was chosen because the fluid was transitioning from the fluid routing layer that is positioned above the conjugate layer. For applications that may require a greater quantity, the reagents could be deposited on both the top and bottom side. Further 
testing would be required to determine the effectiveness of reagent deposition on both sides of the membrane.
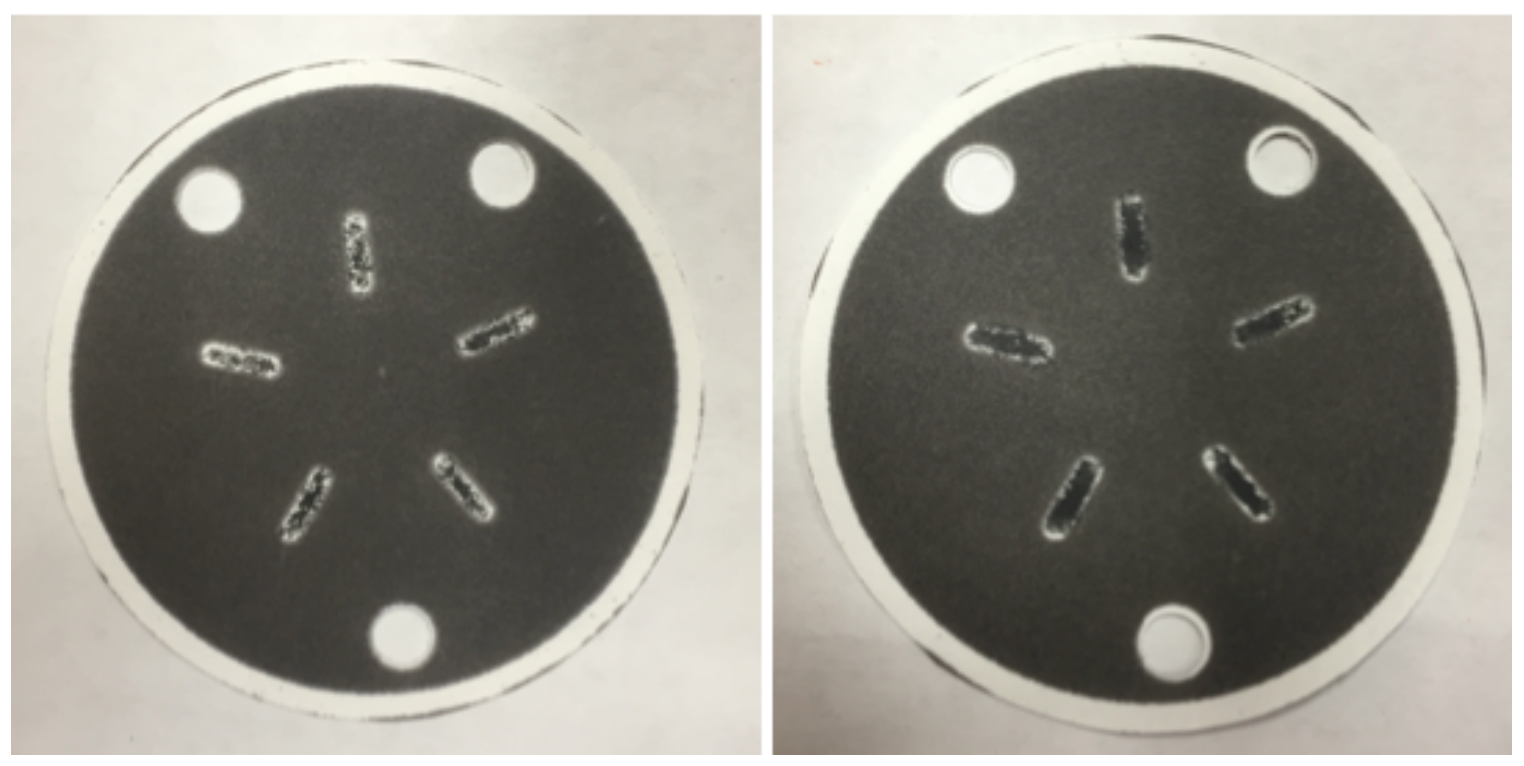

Figure 27: Reagents deposited via a reagent pencil in low (left) and high (right) quantities in the conjugate zones.

It was observed that the leading edge of the fluid, which was clear in appearance, would precede the appearance of a visual signal (Fig. 28). In some tests, the fluid edge would reach the end of the control zone with no visual signal present. However, after a few minutes the blue-green signal would begin to appear in the test zone and continue to advance in the control zone. It was noticed that when the zones of the conjugate layer were colored in more completely with the reagent pen that the signal indicating the presence of glucose would appear in a shorter amount of time. In test 3 and 4 the visual signal was noticed almost immediately. 


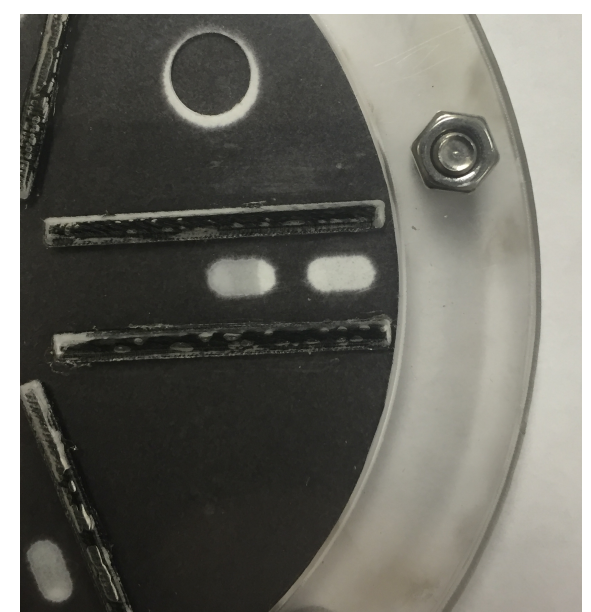

Figure 28: The leading edge of the fluid during the glucose concentration test.

In all tests, the signal was faint when it first appeared and gradually began to intensify as time went on. There was variation in when the signal would reach its peak intensity however; in general, the signal appeared to remain the same after 15 minutes. Figure 29 shows the signal intensity after 5 minutes while Figure 30 shows the signal intensity after 15 minutes.

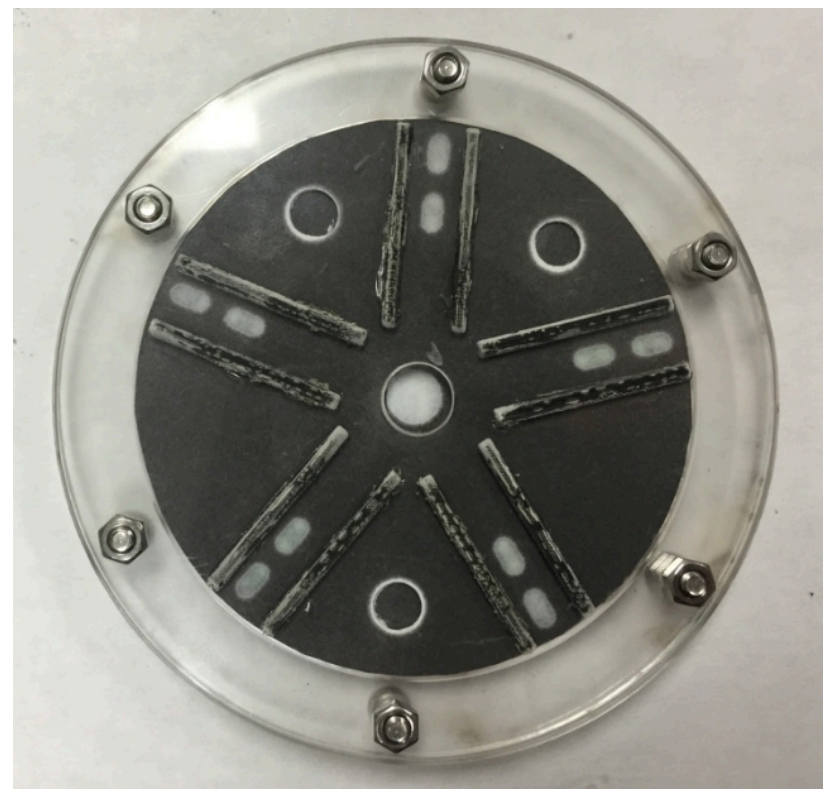

Figure 29: Signal intensity from the $1.2 \mathrm{mM}$ glucose concentration test after 5 minutes. 


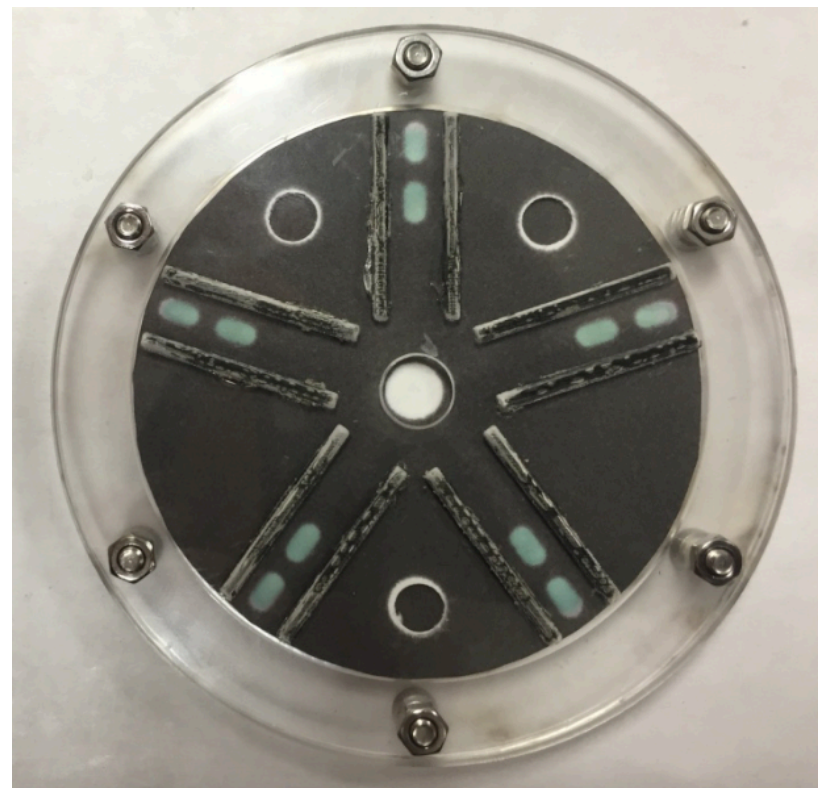

Figure 30: Signal intensity from the $1.2 \mathrm{mM}$ glucose concentration test after 5 minutes.

Additionally, it was observed that the first test conducted resulted in a less intense signal in the test/control zones than the subsequent tests. It is thought that the presence of an oxidation layer on the reagent pencil may have contributed to the lack of signal. Prior to the first test, the reagent pencil had not been used for over a month. Although the outer layer of the pencil tip was rubbed off on a scrap piece of paper before being used, it may not have been sufficient enough for the first test. This suggests that a protocol for removing the oxidation layer may be useful for assuring accurate tests when the reagent pencil has not been used for an extended period of time.

Overall, the outcome of the glucose testing was considered a success. The ability of the device to detect glucose at a concentration of $1.2 \mathrm{mM}$ shows promise for this device as a diagnostic platform for the simultaneous detection of multiple targets. Additionally, the success of the reagent pencil with a multi-layer microPAD opens up more possibilities for future applications of this technology. 


\section{CONCLUSION}

Overall, the primary goal and specific aims of this thesis were fulfilled. The experiments performed showed that rubber cord stock could be used to apply pressure along the edge of the channels that would allow for fluid conductivity across multiple layers. The designs put forth in this thesis demonstrated the ability to rout fluid simultaneously through five separate channels.

The dye tests demonstrated the ability of channel networks and housing units to rout fluid from the sample input to the detection zones. The fluid was seen to move in a consistent manner through each channel. The development times of the successful tests indicate that the device is capable of routing fluid through the device in a timely manner. The final design selected in this thesis was shown to produce successful fluid routing in all channels, well within the desired time limit. This was a major improvement over the previous work as it was the first time successful development was recorded in every channel.

Additionally, it was shown that final microPAD design could successfully function with the reagent pencil created by Martinez et al. By successful detecting glucose from a sample solution, this device has demonstrated the ability to detect a specific analyte from a solution. The colorimetric assay used required no additional equipment to produce a visual signal and demonstrated the potential of this device to be used in a non-laboratory setting. 


\section{FUTURE WORK}

The channel designs and housing units presented in this work are just a few examples of what can be created. The devices that were presented were capable of handling a very large amount of sample input. Future work could look into creating a similar device of a smaller size. A more compact design would allow for less materials used and reduce the overall cost. While the pressure application presented in this work was successful, it may also be beneficial to investigate how applying pressure at different distances from the edge channels would affect the fluid flow. More specifically, future work could look at how the fluid responds when the rubber cord stock overlaps a portion

of the channel. The ability to apply pressure in multiple ways offers more freedom when designing channel layouts.

Since the results from the dye testing indicated there was a significant difference in channel development times, more work would need to be performed to determine the cause. Adjusting the positions of the screw holes so that there they are equally spaced with respect to the edges of the channels is worth investigating. Additionally, a more uniform process of bonding the rubber cord stock to the housing unit should be used to ensure equal pressure distribution between channels.

While the device was tested with an enzymatic glucose assay, it was originally intended to be tested with an antibody or aptamer based assay. Since there was no immobilized detection reagent in the glucose assay the signal was able to flow freely. Future channel designs should take into account the inability to immobilize the signal and 
design the channels such that as much as possible of the signal is concentrated in a visible area. 


\section{BIBLIOGRAPHY}

[1] H. L. Alakomi and M. Saarela, "Salmonella importance and current status of detection and surveillance methods," Quality Assurance and Safety of Crops and Foods, vol. 1, pp. 142-152, 2009.

[2] R. B. Chalker and M. J. Blaser, "A review of human salmonellosis: III. Magnitude of Salmonella infection in the United States," Review of Infectious Diseases, vol. 10, pp. 11-124, 1988.

[3] B. Corburn, G. A. Grassi, and B. B. Finlay, "Salmonella, the host and disease: a brief review," Immunology and Cell Biology, vol. 85, pp. 112-118, 2007.

[4] Germano Mucchetti, Barbara Bonvini, Salvatore Francolino, Erasmo Neviani, and Domenico Carminati, "Effect of washing with a high pressure water spray on removal of Listeria innocua from Gorgonzola cheese rind," Food Control, vol. 19, pp. 521-525, 2008.

[5] D. Kay et al., "Quantification and control of microbial pollution from agriculture: a new policy challenge," Environmental Science \& Policy, vol. 11, pp. 171-184, 2008.

[6] Elaine Scallan et al., "Foodborne Illness Acquired in the United States - Major Pathogens," Emerging Infection Diseases, vol. 17, pp. 7-15, 2011.

[7] Paul D. Frenzen et al., "Salmonella Cost Estimate Updated Using FoodNet Data," FoodReview, vol. 22, pp. 10-15, 1999.

[8] World Health Organization (WHO). (2013, August) Salmonella (non-typhoidal). [Online]. www.who.int/mediacentre/factsheets/fs139/en/

[9] Centers for Disease Control and Prevention (CDC), "An Atlas of Salmonella in the United States, 1968-2011," Altanta, Laboratory-based Enteric Disease Surveillance 2013.

[10] Lay JC, Hand S, Crump JA, Campbell J, Van Duyne MS, Bishop R, Middendor R, Currier M, Mead PS, Mølbak K. Srikantiah P, "Salmonella enterica serotype Javiana infections associated with amphibian contact, Mississippi, 2001," Epidemiol Infect., vol. 132, pp. 273-281, 2004. 
[11] Mick Runyon, Timothy J. Herrman, Robert Phillips, John Hsieh Kyung-Min Lee, "Review of Salmonella detection and identification methods: Aspects of rapid emergency response and food safety," Food Control, vol. 47, pp. 264-276, 2015.

[12] J.L. McKillip K.S. Gracias, "A review of conventional detection and enumeration methods for pathogenic bacteria in food," Canadian Journal of Microbiology, vol. 50, pp. 883-890, 2004.

[13] P. Herrera, F.T. Jones, S.D. Pillai, S.C. Ricke K.G. Maciorowski, "Cultural and immunological detection for Salmonella spp. in animal feeds - a review," Veterinary Research Communications, vol. 30, pp. 127-137, 2006.

[14] R. Naravaneni and K. Jamil, "Rapid detection of food-borne pathogens by using molecular techniques," Journal of Medical Microbiology, vol. 54, pp. 51-54, 2005.

[15] B. Swaminathan and P. Feng, "Rapid detection of food-borne pathogenic bacteria," Annual Reviews in Microbiology, vol. 48, pp. 401-426, 1994.

[16] M. Manafi, "New developments in chromogenic and fluorogenic culture media," International Journal of Food Microbiology, vol. 60, pp. 205-218, 2000.

[17] A. W. Martinez, S. T. Phillips, M. J. Butte, and G. M. Whitesides, "Patterned paper as a platform for inexpensive, low-volume, portable bioassays.," Angew Chem Int Ed Engl., vol. 46, pp. 1318-1320, 2007.

[18] Andres W. Martinez, Scott T. Phillips, and George M. Whiteside, "Diagnostics for the Developing World: Microfluidic Paper-Based Analytical Devices," Anal.Chem., vol. 82, pp. 3-10, 2010.

[19] Jana C. Jokerst et al., "Development of a Paper-Based Analytical Device for Colorimetric Detection of Select Foodborne Pathogens," Anal. Chem., vol. 84, pp. 2900-2907, 2012.

[20] E. Carrilho, S. T. Phillips, S. J. Vella, A W Martinez, and G. M. Whitesides, "Paper microzone plates.," Anal. Chem., vol. 81, pp. 5590-5598, 2009.

[21] Andres W. Martinez, Scott T. Phillips, and George M. Whitesides, "Threedimensional microfluidic devices fabricated in layered paper and tape," Proc Natl Acad Sci U S A, vol. 105, pp. 19606-19611, 2008.

[22] Millipore Corporation, Rapid Lateral Flow Test Strips Considerations for Product Development, 2008. 
[23] Muhammad Sajid, Abdel-Nasser Kawde, and Muhammed Daud, "Designs, formats and applications of lateral flow assay: A literature review," Journal of Saudi Chemical Society, vol. 19, pp. 689-705, 2015.

[24] Jennifer Ward, Nitrocellulose paper based microfluidic platform development and surface functionalization with anti-IgE aptamers , 2012.

[25] Mackenzie Tageson, FUNCTIONAL 3-D CELLULOSE \& NITROCELLULOSE PAPER-BASED, MULITPLEX DIAGNOSTIC PLATFORMS WITHOUT COUPLING AGENTS, 2013.

[26] Zhuangqiang Gao, Li Hou, Mingdi Xu, and Dianping Tang, "Enhanced Colorimetric Immunoassay Accompanying with Enzyme Cascade Amplification Strategy for Ultrasensitive Detection of Low-Abundance Protein," Scientific Reports, vol. 4, p. 3966 EP, 2014.

[27] Weian, et al. Zhao, "Paper-Based Bioassays Using Gold Nanoparticle Colorimetric Probes. ," Analytical Chemistry, pp. 8431-8437, 2008.

[28] L. Anfossi, F. Di Nardo, C. Giovannoli, C. Passini, and C. Baggiani, "Increased sensitivity of lateral flow immunoassay for ochratoxin A through silver enhancement ," Anal Bioanal Chem, vol. 405, pp. 9859-9867, 2013.

[29] Petra Wolffs, Kari Glencross, Romain Thibaudeau, and Mansel Griffiths , "Direct Quantitation and Detection of Salmonellae in Biological Samples without Enrichment, Using Two-Step Filtration and Real-Time PCR," American Society for Microbiology, vol. 72, pp. 3896-3900, 2006.

[30] Zhiyuan Fang, Wei Wu, Xuewen Lu, and Lingwen Zeng , " Lateral flow biosensor for DNA extraction-free detection of salmonella based on aptamer mediated strand displacement amplification," Biosensors and Bioelectronics , vol. 56, pp. 192-197, 2014.

[31] A. H. Free, E. C. Adams, M. L. Kercher, H. M. Free, and M. H. Cook, "Simple specific test for urinary glucose," Clinical Chemistry, vol. 3, pp. 163-168, 1957.

[32] Jinseok Heo and Susan Z. Hua, "An Overview of Recent Strategies in Pathogen Sensing," Sensors, vol. 9, pp. 4483-4502, 2009.

[33] K. L. Josephson, C. P. Gerba, and I. L. Pepper, "Polymerase chain reaction detection of nonviable bacterial pathogens.," Appl Environ Microbiol., vol. 59, pp. 3513-3515, 1993. 